\title{
Unique weak solutions of the d-dimensional micropolar equation with fractional dissipation
}

\author{
Oussama Ben Said ${ }^{* 1}$ and Jiahong $\mathrm{Wu}^{\dagger 2}$ \\ ${ }^{1,2}$ Department of Mathematics, Oklahoma State University, Stillwater, OK \\ 74078, USA
}

December 2, 2019

\begin{abstract}
This article examines the existence and uniqueness of weak solutions to the ddimensional micropolar equations $(d=2$ or $d=3)$ with general fractional dissipation $(-\Delta)^{\alpha} u$ and $(-\Delta)^{\beta} w$. The micropolar equations with standard Laplacian dissipation model fluids with microstructure. The generalization to include fractional dissipation allows simultaneous study of a family of equations and is relevant in some physical circumstances. We establish that, when $\alpha \geq \frac{1}{2}$ and $\beta \geq \frac{1}{2}$, any initial data $\left(u_{0}, w_{0}\right)$ in the critical Besov space $u_{0} \in B_{2,1}^{1+\frac{d}{2}-2 \alpha}\left(\mathbb{R}^{d}\right)$ and $w_{0} \in B_{2,1}^{1+\frac{d}{2}-2 \beta}\left(\mathbb{R}^{d}\right)$ yields a unique weak solution. For $\alpha \geq 1$ and $\beta=0$, any initial data $u_{0} \in B_{2,1}^{1+\frac{d}{2}-2 \alpha}\left(\mathbb{R}^{d}\right)$ and $w_{0} \in B_{2,1}^{\frac{d}{2}}\left(\mathbb{R}^{d}\right)$ also leads to a unique weak solution as well. The regularity indices in these Besov spaces appear to be optimal and can not be lowered in order to achieve the uniqueness. Especially, the 2D micropolar equations with the standard Laplacian dissipation, namely $\alpha=\beta=1$ have a unique weak solution for $\left(u_{0}, w_{0}\right) \in B_{2,1}^{0}$. The proof involves the construction of successive approximation sequences and extensive a priori estimates in Besov space settings.
\end{abstract}

Key words: Micropolar equations, Littlewood-Paley, Local solution, Uniqueness. MSC-2010: 35A01, 35A02, 35Q35, 76D03

\section{Introduction}

The micropolar equations were first proposed in 1965 by C.A Eringen to modal micropolar fluids which are fluids with microstructure (see $[6,10-12])$. These equations can model large

*obensai@okstate.edu

†jiahong.wu@okstate.edu 
number of complex fluids such as animal blood, suspensions, and liquid crystals. In this paper, we focus on the following d-dimensional $(d=2$ or $d=3$ ) incompressible micropolar equations with fractional dissipation

$$
\left\{\begin{array}{l}
\partial_{t} u+(\nu+k)(-\Delta)^{\alpha} u+u \cdot \nabla u+\nabla \Pi-2 k \nabla \times w=0 \\
\partial_{t} w+4 k w+2 k \nabla \times u+u \cdot \nabla w+\gamma(-\Delta)^{\beta} w=0 \\
\nabla \cdot u=0 \\
u(x, 0)=u_{0}(x), \quad w(x, 0)=w_{0}(x)
\end{array}\right.
$$

where $u=u(x, t) \in \mathbb{R}^{d}$ denotes the fluid velocity, $w=w(x, t) \in \mathbb{R}^{d}$ the field of microrotation representing the angular velocity of the rotation of the fluid particles, $\Pi=\Pi(x, t)$ the scalar pressure, and the parameter $\nu$ denotes the Newtonian kinematic viscosity, $k$ the microrotation viscosity and $\gamma$ the angular viscosity. Here the fractional Laplacian operator $(-\Delta)^{\alpha}$ (which is also referred as the Riesz potential operator) is defined via the Fourrier transform

$$
\widehat{(-\Delta)^{\alpha}} f(\xi)=|\xi|^{2 \alpha} \widehat{f}(\xi)
$$

where

$$
\widehat{f}(\xi)=\frac{1}{(2 \pi)^{\frac{d}{2}}} \int_{\mathbb{R}^{d}} e^{-i x \cdot \xi} f(x) d x .
$$

Besides their many physical applications, the micropolar equations are also of great interest in mathematics. Fundamental issues such as the well-posedness problem on (1.1) have recently attracted considerable interest and an array of important results have been established (see, e.g., [2,4,5,13,17-19,21,22,24]). More recent focuses have been on the micropolar equations with partial or fractional dissipation (see, e.g., [7-9, 14, 16, 23]). Investigations on nonlocal diffusion have now become a trend [3]. The study of fractionally dissipated micropolar equations allow us to simultaneously treat a family of equations including those with the standard Laplacian dissipation. The investigations on the micropolar equations with fractional dissipation help reveal how the global well-posedness problem is related to the fractional regularization.

The main goal of this study is to obtain the existence and uniqueness of solutions to (1.1) in a weakest possible functional setting for the largest possible ranges of $\alpha$ and $\beta$. Our main results can be stated as follows.

Theorem 1.1. Consider (1.1) with $\alpha \geq \frac{1}{2}$ and $\beta \geq \frac{1}{2}$. Assume the initial data $u_{0}$ and $w_{0}$ satisfy

$$
\nabla \cdot u_{0}=0, \quad u_{0} \in B_{2,1}^{\frac{d}{2}+1-2 \alpha}\left(\mathbb{R}^{d}\right), \quad w_{0} \in B_{2,1}^{\frac{d}{2}+1-2 \beta}\left(\mathbb{R}^{d}\right) .
$$

Then there exist $T>0$ and a unique weak solution $(u, w)$ of (1.1) on $[0, T]$ satisfying

$$
u \in L^{\infty}\left(0, T, B_{2,1}^{1+\frac{d}{2}-2 \alpha}\left(\mathbb{R}^{d}\right)\right) \cap L^{1}\left(0, T, B_{2,1}^{1+\frac{d}{2}}\left(\mathbb{R}^{d}\right)\right),
$$




$$
w \in L^{\infty}\left(0, T, B_{2,1}^{1+\frac{d}{2}-2 \beta}\left(\mathbb{R}^{d}\right)\right) \cap L^{1}\left(0, T, B_{2,1}^{1+\frac{d}{2}}\left(\mathbb{R}^{d}\right)\right) .
$$

Here $B_{p, q}^{r}$ denotes the inhomogeneous Besov space. A review of the Besov spaces and related facts is provided in the following section. As a special consequence of Theorem 1.1, the two-dimensional (2D) micropolar equations with $\alpha=\beta=1$, namely the standard Laplacian dissipation always possess a unique local solution $(u, w)$ in the critical Besov space $L^{\infty}\left(0, T ; B_{2,1}^{0}\left(\mathbb{R}^{2}\right)\right)$. For the 3D micropolar equations with the standard Laplacian dissipation, the uniqueness is also attained in the critical Besov space $L^{\infty}\left(0, T ; B_{2,1}^{\frac{1}{2}}\left(\mathbb{R}^{3}\right)\right)$. Here the critical Besov spaces are the Besov space settings for which the solution of the differential equations and its scaling invariant counterparts share the same norm. In the general fractional dissipation cases, the regularity indices $1+\frac{d}{2}-2 \alpha$ and $1+\frac{d}{2}-2 \beta$ in the Besov spaces appear to be optimal and one may not be able to achieve the uniqueness when they are lowered.

Theorem 1.2. Consider (1.1) with $\alpha \geq 1$ and $\beta=0$. Assume the initial data $\left(u_{0}, w_{0}\right)$ satisfies

$$
\nabla \cdot u_{0}=0, \quad u_{0} \in B_{2,1}^{1+\frac{d}{2}-2 \alpha}\left(\mathbb{R}^{d}\right), \quad w_{0} \in B_{2,1}^{\frac{d}{2}}\left(\mathbb{R}^{d}\right) .
$$

Then there exist $T>0$ and a unique weak solution $(u, w)$ of (1.1) on $[0, T]$ satisfying

$$
\begin{aligned}
& u \in L^{\infty}\left(0, T, B_{2,1}^{1+\frac{d}{2}-2 \alpha}\left(\mathbb{R}^{d}\right)\right) \cap L^{1}\left(0, T, B_{2,1}^{1+\frac{d}{2}}\left(\mathbb{R}^{d}\right)\right), \\
& w \in L^{\infty}\left(0, T, B_{2,1}^{\frac{d}{2}}\left(\mathbb{R}^{d}\right)\right) .
\end{aligned}
$$

Theorem 1.2 deals with the case when the equation of $w$ involves no diffusion. The Besov space for $u$ remains the same, but the setting for $w$ needs to be in a more regular Besov space due to the lack of diffusion in the equation of $w$. For an inviscid equation, the regularity index $\frac{d}{2}$ in the Besov space $B_{2,1}^{\frac{d}{2}}\left(\mathbb{R}^{d}\right)$ can not be lowered in order to obtain the uniqueness of solutions.

The proof for each of the theorems is naturally split into two parts: the existence and uniqueness parts. The existence part starts with the construction a successive approximation sequence which iteratively solves systems close to (1.1). This successive approximation sequence is then shown to be uniformly bounded in suitable Besov spaces via the method of mathematical induction. These bounds allow us to extract a subsequence, which converges weakly to a limit. Using the Aubin-Lions Lemma, the weak limit is then shown to be the weak solution of (1.1). The main efforts are devoted to proving the uniform boundedness. This process involves various analysis tools and techniques. The uniqueness is established by analyzing the differences in the $L^{2}$ space.

The rest of this paper is divided into three sections. The second section serves as a preparation. It reviews the Besov space and related tools to be used in the subsequent sections. The third section proves Theorem 1.1. It is further divided into two subsections with one devoted to the existence and the other to the uniqueness. The last section provides the proof of Theorem 1.2. It is again split into two subsections, one for the proof of existence and one for the uniqueness. 


\section{Preparations: Besov spaces}

This section serves as a preparation. Materials presented here will be used in the proofs of Theorems 1.1 and 1.2. The definition of the Besov space and related simple facts can be found in [1]. Lemma 2.6 is taken from [15, Lemma A.5]. In what follows, $\mathcal{S}\left(\mathbb{R}^{d}\right)$ denotes the Schwartz class and $\mathcal{S}^{\prime}\left(\mathbb{R}^{d}\right)$ the tempered distribution.

Definition 2.1 (Inhomogenous Besov space $B_{p, q}^{s}$ ). $f \in \mathcal{S}^{\prime}\left(\mathbb{R}^{d}\right)$ belongs to $B_{p, q}^{s}$ with $s \in \mathbb{R}$ and $1 \leq p \leq q \leq \infty$ if

$$
\|f\|_{B_{p, q}^{s}} \equiv\left\|2^{s j}\right\| \Delta_{j} f\left\|_{L^{p}}\right\|_{l^{q}}= \begin{cases}\left(\sum_{j=-1}^{+\infty}\left(2^{s j}\left\|\Delta_{j} f\right\|_{L^{p}}\right)^{q}\right)^{\frac{1}{q}} & \text { if } \\ \sup _{j \geq-1} 2^{s j}\left\|\Delta_{j} f\right\|_{L^{p}} & \text { if } q=\infty,\end{cases}
$$

is finite.

Lemma 2.2. Let $B(0, r)$ and $C\left(0, r_{1}, r_{2}\right)$ denote the standard ball and the annulus, respectively,

$$
B(0, r)=\left\{\xi \in \mathbb{R}^{d},|\xi| \leq r\right\}, \quad C\left(0, r_{1}, r_{2}\right)=\left\{\xi \in \mathbb{R}^{d}, \quad r_{1} \leq|\xi| \leq r_{2}\right\}
$$

There are two compactly supported smooth radial functions $\phi$ and $\psi$ satisfying

$$
\begin{aligned}
& \operatorname{supp} \phi \subset B\left(0, \frac{4}{3}\right), \quad \operatorname{supp} \psi \subset C\left(0, \frac{3}{4}, \frac{8}{3}\right), \\
& \phi(\xi)+\sum_{j \geq 0} \psi\left(2^{-j} \xi\right)=1 \quad \text { for all } \xi \in \mathbb{R}^{d} .
\end{aligned}
$$

The proof of Lemma 2.2 can be found in [1, p.59].

Notations 2.2.1. We use $\widetilde{h}$ and $h$ to denote the inverse Fourier transforms of $\phi$ and $\psi$ respectively

$$
\widetilde{h}=\mathcal{F}^{-1} \phi, \quad h=\mathcal{F}^{-1} \psi
$$

We write $\psi_{j}(\xi)=\psi\left(2^{-j} \xi\right)$. By a simple property of the Fourier transform,

$$
h_{j}(x):=\mathcal{F}^{-1} \psi_{j}(x)=2^{d j} h\left(2^{j} x\right) .
$$

Definition 2.3. The inhomogeneous dyadic block operator $\Delta_{j}$ are defined as

$$
\begin{array}{ll}
\Delta_{j} f=0 & \text { for } j \leq-2, \\
\Delta_{-1} f=\widetilde{h} * f=\int_{\mathbb{R}^{d}} f(x-y) \widetilde{h}(y) d y, &
\end{array}
$$




$$
\Delta_{j} f=h_{j} * f=2^{d j} \int_{\mathbb{R}^{d}} f(x-y) h\left(2^{j} y\right) d y \quad \text { for } j \geq 0
$$

The corresponding inhomogeneous low frequency cut-off operator $S_{j}$ is defined by

$$
S_{j} f=\sum_{k \leq j-1} \Delta_{k} f
$$

Remarks 2.3.1. For any function $f$ in the usual Schwarz class $\mathcal{S}$, (2.1) implies

$$
\widehat{f}(\xi)=\phi(\xi) \widehat{f}(\xi)+\sum_{j \geq 0} \psi\left(2^{-j} \xi\right) \widehat{f}(\xi)
$$

or in terms of the inhomogenous dyadic block operators

$$
f=\sum_{j \geq-1} \Delta_{j} f \quad \text { or } \quad I d=\sum_{j \geq-1} \Delta_{j},
$$

where Id denotes the identity operator. For generality, for any $F$ in the space of tempered distributions $\mathcal{S}^{\prime}$,

$$
F=\sum_{j \geq-1} \Delta_{j} F \quad \text { or } \quad I d=\sum_{j \geq-1} \Delta_{j} \quad \text { in } \quad \mathcal{S}^{\prime}
$$

(2.2) is referred to as the Littlewood-Paley decomposition for tempered distributions.

Definition 2.4. In terms of inhomogeneous dyadic block operators, we can write the standard product in terms of the paraproducts, namely

$$
F G=\sum_{|j-k|<2} S_{k-1} F \Delta_{k} G+\sum_{|j-k|<2} \Delta_{k} F S_{k-1} G+\sum_{k \geq j-1} \Delta_{k} F \widetilde{\Delta}_{k} G,
$$

where $\widetilde{\Delta}_{k}=\Delta_{k-1}+\Delta_{k}+\Delta_{k+1}$. This is the so-called Bony decomposition.

Lemma 2.5. Let $\alpha \geq 0$. Let $1 \leq p \leq q \leq \infty$.

(1) If $f$ satisfies

$$
\operatorname{supp} \tilde{f} \subset\left\{\xi \in \mathbb{R}^{d}, \quad|\xi| \leq K 2^{j}\right\},
$$

for some integer $j$ and a constant $K>0$ then

$$
\left\|(-\Delta)^{\alpha} f\right\|_{L^{q}\left(\mathbb{R}^{d}\right)} \leq c_{1} 2^{2 \alpha j+j d\left(\frac{1}{p}-\frac{1}{q}\right)}\|f\|_{L^{p}\left(\mathbb{R}^{d}\right)} .
$$

(2) If $f$ satisfies

$$
\operatorname{supp} \tilde{f} \subset\left\{\xi \in \mathbb{R}^{d}, \quad K_{1} 2^{j} \leq|\xi| \leq K_{2} 2^{j}\right\},
$$

for some integer $j$ and constants $0<K_{1} \leq K_{2}$ then

$$
c_{1} 2^{2 \alpha j}\|f\|_{L^{q\left(\mathbb{R}^{d}\right)}} \leq\left\|(-\Delta)^{\alpha} f\right\|_{L^{q\left(\mathbb{R}^{d}\right)}} \leq c_{2} 2^{2 \alpha j+j d\left(\frac{1}{p}-\frac{1}{q}\right)}\|f\|_{L^{p}\left(\mathbb{R}^{d}\right)},
$$

where $c_{1}, c_{2}$ are constants depending only on $\alpha, p, q$. 
Below we state bounds for the triple products involving Fourier localized functions. These bounds will be used in the proofs of Theorems 1.1 and 1.2. We refer the reader to Lemma A.5 in [15] for a detailed proof of the following lemma.

Lemma 2.6. Let $j \geq 0$ be an integer. Let $\Delta_{j}$ be the inhomogeneous Littlewood-Paleylocalization operator. For any vectors field $F, G, H$ with $\nabla \cdot F=0$ we have

$$
\begin{gathered}
\left|\int_{\mathbb{R}^{d}} \Delta_{j}(F \cdot \nabla G) \cdot \Delta_{j} H d x\right| \leq c\left\|\Delta_{j} H\right\|_{L^{2}}\left(2^{j} \sum_{m \leq j-1} 2^{\frac{d}{2} m}\left\|\Delta_{m} F\right\|_{L^{2}} \sum_{|j-k| \leq 2}\left\|\Delta_{k} G\right\|_{L^{2}}\right. \\
\left.\quad+\sum_{|j-k| \leq 2}\left\|\Delta_{k} F\right\|_{L^{2}} \sum_{m \leq j-1} 2^{\left(1+\frac{d}{2}\right) m}\left\|\Delta_{m} G\right\|_{L^{2}}+\sum_{k \leq j-1} 2^{j} 2^{\frac{d}{2} k}\left\|\Delta_{k} F\right\|_{L^{2}}\left\|\widetilde{\Delta}_{k} G\right\|_{L^{2}}\right)
\end{gathered}
$$

and

$$
\begin{aligned}
& \left|\int_{\mathbb{R}^{d}} \Delta_{j}(F \cdot \nabla G) \cdot \Delta_{j} G d x\right| \leq c\left\|\Delta_{j} G\right\|_{L^{2}}\left(\sum_{m \leq j-1} 2^{\left(1+\frac{d}{2}\right) m}\left\|\Delta_{m} F\right\|_{L^{2}} \sum_{|j-k| \leq 2}\left\|\Delta_{k} G\right\|_{L^{2}}\right. \\
& \left.\quad+\sum_{|j-k| \leq 2}\left\|\Delta_{k} F\right\|_{L^{2}} \sum_{m \leq j-1} 2^{\left(1+\frac{d}{2}\right) m}\left\|\Delta_{m} G\right\|_{L^{2}}+\sum_{k \leq j-1} 2^{j} 2^{\frac{d}{2} k}\left\|\Delta_{k} F\right\|_{L^{2}}\left\|\widetilde{\Delta}_{k} G\right\|_{L^{2}}\right) .
\end{aligned}
$$

\section{Proof of Theorem 1.1}

\subsection{Existence of a weak solution}

This subsection proves the existence part of Theorem 1.1. The approach is to construct a successive approximation sequence and show that the limit of a subsequence actually solves (1.1) in the weak sense.

Proof for the existence part of Theorem 1.1. We consider a successive approximation $\left\{\left(u^{(n)}, w^{(n)}\right)\right\}$ satisfying

$$
\left\{\begin{array}{l}
u^{(1)}=S_{2} u_{0}, \quad w^{(1)}=S_{2} w_{0}, \\
\partial_{t} u^{(n+1)}+(\nu+k)(-\Delta)^{\alpha} u^{(n+1)}=\mathbb{P}\left(-u^{(n)} \cdot \nabla u^{(n+1)}\right)+2 k \nabla \times w^{(n)}, \\
\partial_{t} w^{(n+1)}+\gamma(-\Delta)^{\beta} w^{(n+1)}=-4 k w^{(n+1)}-2 k \nabla \times u^{(n)}-u^{(n)} \cdot \nabla w^{(n+1)}, \\
u^{(n+1)}(x, 0)=S_{n+1} u_{0}, \quad w^{(n+1)}(x, 0)=S_{n+1} w_{0},
\end{array}\right.
$$

where $\mathbb{P}=I-\nabla(-\Delta)^{-1}$ div is the standard Leray Projection. For

$$
M=2\left(\left\|u_{0}\right\|_{B_{2,1}^{1+\frac{d}{2}-2 \alpha}}+\left\|w_{0}\right\|_{B_{2,1}^{1+\frac{d}{2}-2 \beta}}\right),
$$


$T>0$ sufficiently small and $0<\delta<1$ (to be specified later), we set

$$
\begin{gathered}
Y \equiv\left\{(u, w) \mid\|u\|_{L^{\infty}\left(0, T, B_{2,1}^{1+\frac{d}{2}-2 \alpha}\right)} \leq M,\|w\|_{L^{\infty}\left(0, T, B_{2,1}^{1+\frac{d}{2}-2 \beta}\right)} \leq M,\right. \\
\left.\|u\|_{L^{1}\left(0, T, B_{2,1}^{1+\frac{d}{2}}\right)} \leq \delta,\|w\|_{L^{1}\left(0, T, B_{2,1}^{1+\frac{d}{2}}\right)} \leq \delta\right\} .
\end{gathered}
$$

We show that $\left\{\left(u^{(n)}, w^{(n)}\right)\right\}$ has a subsequence that converges to the weak solution of (1.1). This process consists of three main steps. The first step is to show that $\left\{\left(u^{(n)}, w^{(n)}\right)\right\}$ is uniformly bounded in $Y$. The second step is to extract a strongly convergent subsequence via the Aubin-Lions Lemma. While the last step is to show that the limit is indeed a weak solution of (1.1).

To show the uniform bound for $\left\{\left(u^{(n)}, w^{(n)}\right)\right\}$ in Y, we prove by induction. Clearly,

$$
\begin{aligned}
& \left\|u^{(1)}\right\|_{L^{\infty}\left(0, T, B_{2,1}^{1+\frac{d}{2}-2 \alpha}\right)}=\left\|S_{2} u_{0}\right\|_{L^{\infty}\left(0, T, B_{2,1}^{1+\frac{d}{2}-2 \alpha}\right)} \leq M, \\
& \left\|w^{(1)}\right\|_{L^{\infty}\left(0, T, B_{2,1}^{1+\frac{d}{2}-2 \beta}\right)}=\left\|S_{2} w_{0}\right\|_{L^{\infty}\left(0, T, B_{2,1}^{1+\frac{d}{2}-2 \beta}\right)} \leq M .
\end{aligned}
$$

If $T>0$ is sufficiently small, then

$$
\begin{gathered}
\left\|u^{(1)}\right\|_{L^{1}\left(0, T, B_{2,1}^{1+\frac{d}{2}}\right)} \leq T\left\|S_{2} u_{0}\right\|_{B_{2,1}^{1+\frac{d}{2}}} \leq T c\left\|u_{0}\right\|_{B_{2,1}^{1+\frac{d}{2}-2 \alpha}} \leq \delta, \\
\left\|w^{(1)}\right\|_{L^{1}\left(0, T, B_{2,1}^{1+\frac{d}{2}}\right)} \leq T\left\|S_{2} w_{0}\right\|_{B_{2,1}^{1+\frac{d}{2}}} \leq T c\left\|w_{0}\right\|_{B_{2,1}^{1+\frac{d}{2}-2 \beta}} \leq \delta .
\end{gathered}
$$

Assuming that $\left(u^{(n)}, w^{(n)}\right)$ obeys the bounds defined in $Y$, namely

$$
\begin{aligned}
& \left\|u^{(n)}\right\|_{L^{\infty}\left(0, T, B_{2,1}^{1+\frac{d}{2}-2 \alpha}\right)} \leq M,\left\|w^{(1)}\right\|_{L^{\infty}\left(0, T, B_{2,1}^{1+\frac{d}{2}-2 \beta}\right)} \leq M, \\
& \left\|u^{(n)}\right\|_{L^{1}\left(0, T, B_{2,1}^{1+\frac{d}{2}}\right)} \leq \delta, \quad\left\|w^{(n)}\right\|_{L^{1}\left(0, T, B_{2,1}^{1+\frac{d}{2}}\right)} \leq \delta,
\end{aligned}
$$

we prove that $\left\{\left(u^{(n+1)}, w^{(n+1)}\right)\right\}$ obeys the same bound for suitably selected $T>0, M>0$ and $\delta>0$. For the sake of clarity, the proof of the four bounds in achieved in the following four steps.

3.1.1 The estimate of $u^{(n+1)}$ in $B_{2,1}^{1+\frac{d}{2}-2 \alpha}\left(\mathbb{R}^{d}\right)$. Let $j \geq 0$ be an integer. Applying $\Delta_{j}$ to the second equation in (3.1) and then dotting with $\Delta_{j} u^{(n+1)}$, we obtain

$$
\frac{1}{2} \frac{d}{d t}\left\|\Delta_{j} u^{(n+1)}\right\|_{L^{2}}^{2}+(\nu+k)\left\|\Lambda^{\alpha} \Delta u^{(n+1)}\right\|_{L^{2}}^{2}=A_{1}+A_{2},
$$


where

$$
\begin{aligned}
& A_{1}=\int_{\mathbb{R}^{d}} 2 k \Delta_{j}\left(\nabla \times w^{(n)}\right) \cdot \Delta_{j} u^{(n+1)} d x, \\
& A_{2}=-\int \Delta_{j}\left(u^{(n)} \cdot \nabla u^{(n+1)}\right) \Delta_{j} u^{(n+1)} d x .
\end{aligned}
$$

We remark that the projection operator $\mathbb{P}$ has been eliminated due to the divergence-free condition $\nabla \cdot u^{(n+1)}=0$. The dissipative part admits a lower bound

$$
(\nu+k)\left\|\Lambda^{\alpha} \Delta_{j} u^{(n+1)}\right\|_{L^{2}}^{2} \geq c_{0} 2^{2 \alpha j}\left\|\Delta_{j} u^{(n+1)}\right\|_{L^{2}}^{2},
$$

where $c_{0}>0$ is a constant. By Hölder's inequality and Bernstein's inequality

$$
\begin{aligned}
\left|A_{1}\right| & =\left|\int_{\mathbb{R}^{d}} 2 k \Delta_{j}\left(\nabla \times w^{(n)}\right) \cdot \Delta_{j} u^{(n+1)} d x\right| \\
& \leq 2 k\left\|\Delta_{j}\left(\nabla \times w^{(n)}\right)\right\|_{L^{2}}\left\|\Delta_{j} u^{(n+1)}\right\|_{L^{2}} \\
& \leq c 2^{j}\left\|\Delta_{j} w^{(n)}\right\|_{L^{2}}\left\|\Delta_{j} u^{(n+1)}\right\|_{L^{2}} .
\end{aligned}
$$

According to Lemma 2.6,

$$
\begin{aligned}
\left|A_{2}\right|= & \left|-\int_{\mathbb{R}^{d}} \Delta_{j}\left(u^{(n)} \cdot \nabla u^{(n+1)}\right) \cdot \Delta_{j} u^{(n+1)} d x\right| \\
\leq & c\left\|\Delta_{j} u^{(n+1)}\right\|_{L^{2}}^{2} \sum_{m \leq j-1} 2^{\left(1+\frac{d}{2}\right) m}\left\|\Delta_{m} u^{(n)}\right\|_{L^{2}} \\
& +c\left\|\Delta_{j} u^{(n+1)}\right\|_{L^{2}}\left\|\Delta_{j} u^{(n)}\right\|_{L^{2}} \sum_{m \leq j} 2^{\left(1+\frac{d}{2}\right) m}\left\|\Delta_{m} u^{(n+1)}\right\|_{L^{2}} \\
& +c \sum_{k \geq j-1} 2^{j} 2^{\frac{d}{2} k}\left\|\Delta_{k} u^{(n)}\right\|_{L^{2}}\left\|\widetilde{\Delta}_{k} u^{(n+1)}\right\|_{L^{2}}\left\|\Delta_{j} u^{(n+1)}\right\|_{L^{2}} .
\end{aligned}
$$

Inserting the estimates above in (3.3) and eliminating $\left\|\Delta_{j} u^{(n+1)}\right\|_{L^{2}}$ from the both sides, we obtain

$$
\frac{d}{d t}\left\|\Delta_{j} u^{(n+1)}\right\|_{L^{2}}+c_{0} 2^{2 \alpha j}\left\|\Delta_{j} u^{(n+1)}\right\|_{L^{2}} \leq J_{1}+J_{2}+J_{3}+J_{4},
$$

where

$$
\begin{aligned}
& J_{1}=c\left\|\Delta_{j} u^{(n+1)}\right\|_{L^{2}} \sum_{m \leq j-1} 2^{\left(1+\frac{d}{2}\right) m}\left\|\Delta_{m} u^{(n)}\right\|_{L^{2}}, \\
& J_{2}=c\left\|\Delta_{j} u^{(n)}\right\|_{L^{2}} \sum_{m \leq j} 2^{\left(1+\frac{d}{2}\right) m}\left\|\Delta_{m} u^{(n+1)}\right\|_{L^{2}}, \\
& J_{3}=c 2^{j} \sum_{k \geq j-1} 2^{\frac{d}{2} k}\left\|\widetilde{\Delta}_{k} u^{(n+1)}\right\|_{L^{2}}\left\|\Delta_{k} u^{(n)}\right\|_{L^{2}}, \\
& J_{4}=c 2^{j}\left\|\Delta_{j} w^{(n)}\right\|_{L^{2}} .
\end{aligned}
$$


Integrating (3.4) in time yields

$$
\left\|\Delta_{j} u^{(n+1)}\right\|_{L^{2}} \leq e^{-c_{0} 2^{2 \alpha j} t}\left\|\Delta_{j} u_{0}^{(n+1)}\right\|_{L^{2}}+\int_{0}^{t} e^{-c_{0} 2^{2 \alpha j}(t-\tau)}\left(J_{1}+\cdots+J_{4}\right) d \tau .
$$

Multiplying (3.5) by $2^{\left(1+\frac{d}{2}-2 \alpha\right) j}$ and summing over $j$, we obtain

$$
\left\|u^{(n+1)}(t)\right\|_{B_{2,1}^{1+\frac{d}{2}-2 \alpha}} \leq\left\|u_{0}^{(n+1)}\right\|_{B_{2,1}^{1+\frac{d}{2}-2 \alpha}}+\sum_{j \geq-1} 2^{\left(1+\frac{d}{2}-2 \alpha\right)} \int_{0}^{t} e^{-c_{0} 2^{2 \alpha j}(t-\tau)}\left(J_{1}+\cdots+J_{4}\right) d \tau .
$$

The terms on the right hand side of (3.6) can be estimated as follows using the simple bound

$$
e^{-c_{0} 2^{2 \alpha j}(t-\tau)} \leq 1
$$

Recalling the definition of $J_{1}$ above and using the inductive assumption on $u^{(n)}$, we have for any $t \leq T$,

$$
\begin{aligned}
\sum_{j \geq-1} 2^{\left(1+\frac{d}{2}-2 \alpha\right) j} & \int_{0}^{t} e^{-c_{0} 2^{2 \alpha j}(t-\tau)} J_{1} d \tau \\
& \leq c \int_{0}^{t} \sum_{j \geq-1} 2^{\left(1+\frac{d}{2}-2 \alpha\right) j} \mid \Delta_{j} u^{(n+1)}\left\|_{L^{2}} \sum_{m \leq j-1} 2^{\left(1+\frac{d}{2}\right) m}\right\| \Delta_{m} u^{(n)}(\tau) \|_{L^{2}} d \tau \\
& \leq c \int_{0}^{t}\left\|u^{(n+1)}(\tau)\right\|_{B_{2,1}^{1+\frac{d}{2}-2 \alpha}}\left\|u^{(n)}(\tau)\right\|_{B_{2,1}^{1+\frac{d}{2}}} d \tau \\
& \leq c\left\|u^{(n+1)}\right\|_{L^{\infty}\left(0, t, B_{2,1}^{1+\frac{d}{2}-2 \alpha}\right)}\left\|u^{(n)}\right\|_{L^{1}\left(0, t, B_{2,1}^{1+\frac{d}{2}}\right)}\|\|_{L^{\infty}\left(0, T, B_{2,1}^{1+\frac{d}{2}-2 \alpha}\right)}\left\|u^{(n)}\right\|_{L^{1}\left(0, T, B_{2,1}^{1+\frac{d}{2}}\right)} \leq c \delta\left\|u^{(n+1)}\right\|_{L^{\infty}\left(0, T, B_{2,1}^{1+\frac{d}{2}-2 \alpha}\right)} \\
& \leq c u^{(n+1)} \|
\end{aligned}
$$

The term involving $J_{2}$ admits the same bound. In fact, by Young's inequality for series convolution,

$$
\begin{aligned}
\sum_{j \geq-1} 2^{\left(1+\frac{d}{2}-2 \alpha\right) j} \int_{0}^{t} & e^{-c_{0} 2^{2 \alpha j}(t-\tau)} J_{2} d \tau \\
& \leq c \int_{0}^{t} \sum_{j \geq-1} 2^{\left(1+\frac{d}{2}\right) j}\left\|\Delta_{j} u^{(n)}\right\|_{L^{2}} \sum_{m \leq j} 2^{2 \alpha(m-j)} 2^{\left(1+\frac{d}{2}-2 \alpha\right) m}\left\|\Delta_{m} u^{(n+1)}(\tau)\right\|_{L^{2}} d \tau \\
& \leq c \int_{0}^{t}\left\|u^{(n)}(\tau)\right\|_{B_{2,1}^{1+\frac{d}{2}}}\left\|u^{(n+1)}(\tau)\right\|_{B_{2,1}^{1+\frac{d}{2}-2 \alpha}} d \tau \\
& \leq c\left\|u^{(n)}\right\|_{L^{1}\left(0, T, B_{2,1}^{1+\frac{d}{2}}\right)}\left\|u^{(n+1)}\right\|_{L^{\infty}\left(0, T, B_{2,1}^{1+\frac{d}{2}-2 \alpha}\right)} \leq c \delta\left\|u^{(n+1)}\right\|_{L^{\infty}\left(0, T, B_{2,1}^{1+\frac{d}{2}-2 \alpha}\right)}
\end{aligned}
$$

The estimate for the term with $J_{3}$ is also similar,

$\sum_{j \geq-1} 2^{\left(1+\frac{d}{2}-2 \alpha\right) j} \int_{0}^{t} e^{-c_{0} 2^{2 \alpha j}(t-\tau)} J_{3} d \tau$ 


$$
\begin{aligned}
& =\int_{0}^{t} \sum_{j \geq-1} 2^{\left(1+\frac{d}{2}-2 \alpha\right) j} \sum_{k \geq j-1} c 2^{j} 2^{\frac{d}{2} k}\left\|\widetilde{\Delta}_{k} u^{(n+1)}\right\|_{L^{2}}\left\|\Delta_{k} u^{(n)}\right\|_{L^{2}} d \tau \\
& =c \int_{0}^{t} \sum_{j \geq-1} \sum_{k \geq j-1} 2^{\left(2+\frac{d}{2}-2 \alpha\right)(j-k)} 2^{\left(1+\frac{d}{2}\right) k}\left\|\Delta_{k} u^{(n)}\right\|_{L^{2}} 2^{\left(1+\frac{d}{2}-2 \alpha\right) k}\left\|\widetilde{\Delta}_{k} u^{(n+1)}\right\|_{L^{2}} d \tau \\
& \leq c \int_{0}^{t}\left\|u^{(n)}(\tau)\right\|_{B_{2,1}^{1+\frac{d}{2}}}\left\|u^{(n+1)}(\tau)\right\|_{B_{2,1}^{1+\frac{d}{2}-2 \alpha}} d \tau \\
& \leq c\left\|u^{(n)}\right\|_{L^{1}\left(0, T, B_{2,1}+\frac{d}{2}\right)}\left\|u^{(n+1)}\right\|_{L^{\infty}\left(0, T, B_{2,1}^{1+\frac{d}{2}-2 \alpha}\right)} \leq c \delta\left\|u^{(n+1)}\right\|_{L^{\infty}\left(0, T, B_{2,1}^{1+\frac{d}{2}-2 \alpha}\right)} .
\end{aligned}
$$

It remains to bound the term with $J_{4}$,

$$
\begin{aligned}
\sum_{j \geq-1} 2^{\left(1+\frac{d}{2}-2 \alpha\right) j} \int_{0}^{t} e^{-c_{0} 2^{2 \alpha j}(t-\tau)} J_{4} d \tau & \\
= & \sum_{j \geq-1} 2^{\left(1+\frac{d}{2}-2 \alpha\right) j} \int_{0}^{t} e^{-c_{0} 2^{2 \alpha j}(t-\tau)} c 2^{j}\left\|\Delta_{j} w^{(n)}\right\|_{L^{2}} d \tau \\
\leq & \sum_{j \geq-1} 2^{\left(1+\frac{d}{2}-2 \alpha\right) j} \int_{0}^{t} c 2^{j}\left\|\Delta_{j} w^{(n)}\right\|_{L^{2}} d \tau=c \int_{0}^{t} \sum_{j \geq-1} 2^{\left(2+\frac{d}{2}-2 \alpha\right) j}\left\|\Delta_{j} w^{(n)}\right\|_{L^{2}} d \tau \\
& \leq \int_{0}^{t} \sum_{j \geq-1} 2^{\left(1+\frac{d}{2}\right) j}\left\|\Delta_{j} w^{(n)}\right\|_{L^{2}} d \tau=c\left\|w^{(n)}\right\|_{L^{1}\left(0, t, B_{2,1}^{1+\frac{d}{2}}\right)} \leq c \delta
\end{aligned}
$$

Collecting the bounds above and inserting them in (3.6), we find for any $t \leq T$

$$
\left\|u^{(n+1)}(t)\right\|_{B_{2,1}^{1+\frac{d}{2}-2 \alpha}} \leq\left\|u_{0}^{(n+1)}\right\|_{B_{2,1}^{1+\frac{d}{2}-2 \alpha}}+c \delta\left\|u^{(n+1)}\right\|_{L^{\infty}\left(0, T, B_{2,1}^{1+\frac{d}{2}-2 \alpha}\right)}+c \delta .
$$

Therefore

$$
\left\|u^{(n+1)}(t)\right\|_{L^{\infty}\left(0, T, B_{2,1}^{1+\frac{d}{2}-2 \alpha}\right)} \leq\left\|u_{0}^{(n+1)}\right\|_{B_{2,1}^{1+\frac{d}{2}-2 \alpha}}+c \delta\left\|u^{(n+1)}\right\|_{L^{\infty}\left(0, T, B_{2,1}^{1+\frac{d}{2}-2 \alpha}\right)}+c \delta .
$$

Choosing $\delta$ such that $c \delta \leq \min \left(\frac{1}{4}, \frac{M}{4}\right)$ we get

$$
\left\|u^{(n+1)}(t)\right\|_{L^{\infty}\left(0, T, B_{2,1}^{1+\frac{d}{2}-2 \alpha}\right)} \leq \frac{M}{2}+\frac{1}{4}\left\|u^{(n+1)}\right\|_{L^{\infty}\left(0, T, B_{2,1}^{1+\frac{d}{2}-2 \alpha}\right)}+\frac{M}{4}
$$

which implies

$$
\left\|u^{(n+1)}(t)\right\|_{L^{\infty}\left(0, T, B_{2,1}^{1+\frac{d}{2}-2 \alpha}\right)} \leq M
$$

3.1.2 The estimate of $\left\|u^{(n+1)}\right\|_{L^{1}\left(0, T, B^{1+\frac{d}{2}}\right)}$. We multiply (3.5) by $2^{\left(1+\frac{d}{2}\right) j}$, sum over $j$ and integrate in time to obtain

$$
\left\|u^{(n+1)}\right\|_{L^{1}\left(0, T, B_{2,1}^{1+\frac{d}{2}}\right)} \leq \int_{0}^{T} \sum_{j \geq-1} 2^{\left(1+\frac{d}{2}\right) j} e^{-c_{0} 2^{2 \alpha j} t}\left\|\Delta_{j} u_{0}^{(n+1)}\right\|_{L^{2}} d t
$$




$$
+\int_{0}^{T} \int_{0}^{s} \sum_{j \geq-1} 2^{\left(1+\frac{d}{2}\right) j} e^{-c_{0} 2^{2 \alpha j}(s-\tau)}\left(J_{1}+\cdots+J_{4}\right) d \tau d s
$$

We estimate the terms on the right hand side of (3.7) and start with the first term.

$$
\int_{0}^{T} \sum_{j \geq-1} 2^{\left(1+\frac{d}{2}\right) j} e^{-c_{0} 2^{2 \alpha j} t}\left\|\Delta_{j} u_{0}^{n+1}\right\|_{L^{2}} d t=c \sum_{j \geq-1} 2^{\left(1+\frac{d}{2}-2 \alpha\right) j}\left(1-e^{-c_{0} 2^{2 \alpha j} T}\right)\left\|\Delta_{j} u_{0}^{(n+1)}\right\|_{L^{2}}
$$

Since $u_{0} \in B_{2,1}^{\left(1+\frac{d}{2}-2 \alpha\right)}$, then by Dominated convergence Theorem

$$
\lim _{T \rightarrow 0} \sum_{j \geq-1} 2^{\left(1+\frac{d}{2}-2 \alpha\right) j}\left(1-e^{-c_{0} 2^{2 \alpha j} T}\right)\left\|\Delta_{j} u_{0}^{(n+1)}\right\|_{L^{2}}=0 .
$$

Therefore, we can choose $T$ sufficiently small such that

$$
\int_{0}^{T} \sum_{j \geq-1} 2^{\left(1+\frac{d}{2}\right) j} e^{-c_{0} 2^{2 \alpha j} t}\left\|\Delta_{j} u_{0}^{(n+1)}\right\|_{L^{2}} d t \leq \frac{\delta}{4}
$$

Applying Young's inequality for the time convolution, we have

$$
\begin{aligned}
& \int_{0}^{T} \sum_{j \geq-1} 2^{\left(1+\frac{d}{2}\right) j} \int_{0}^{s} e^{-c_{0} 2^{2 \alpha j}(s-\tau)} J_{1} d \tau d s \\
& \quad=c \int_{0}^{T} \sum_{j \geq-1} 2^{\left(1+\frac{d}{2}\right) j} \int_{0}^{s} e^{-c_{0} 2^{2 \alpha j}(s-\tau)}\left\|\Delta_{j} u^{(n+1)}(\tau)\right\|_{L^{2}} \sum_{m \leq j-1} 2^{\left(1+\frac{d}{2}\right) m}\left\|\Delta_{m} u^{(m)}\right\|_{L^{2}} d \tau d s \\
& \quad \leq c \sum_{j \geq-1} 2^{\left(1+\frac{d}{2}\right) j} \int_{0}^{T}\left\|\Delta_{j} u^{(n+1)}(\tau)\right\|_{L^{2}} \sum_{m \leq j-1} 2^{\left(1+\frac{d}{2}\right) m}\left\|\Delta_{m} u^{(m)}\right\|_{L^{2}} d \tau \int_{0}^{T} e^{-c_{0} 2^{2 \alpha j} s} d s
\end{aligned}
$$

Using the fact that there exists $c_{2}>0$ satisfying for $j \geq 0$,

$$
\int_{0}^{s} e^{-c_{0} 2^{2 \alpha j} s} d s \leq c 2^{-2 \alpha j}\left(1-e^{-c_{2} T}\right)
$$

we get

$$
\begin{aligned}
\int_{0}^{T} \sum_{j \geq-1} 2^{\left(1+\frac{d}{2}\right) j} & \int_{0}^{s} e^{-c_{0} 2^{2 \alpha j}(s-\tau)} J_{1} d \tau d s \\
& \leq c\left(1-e^{-c_{2} T}\right) \int_{0}^{T} \sum_{j} 2^{\left(1+\frac{d}{2}-2 \alpha\right) j}\left\|\Delta_{j} u^{(n+1)}(\tau)\right\|_{L^{2}} \sum_{m \leq j-1} 2^{\left(1+\frac{d}{2}\right) m}\left\|\Delta_{m} u^{(m)}\right\|_{L^{2}} d \tau \\
& \leq c\left(1-e^{-c_{2} T}\right)\left\|u^{(n+1)}\right\|_{L^{\infty}\left(0, T, B_{2,1}^{1+\frac{d}{2}-2 \alpha}\right)} \underbrace{\left\|u^{(n)}\right\|_{L^{1}\left(0, T, B_{2,1}^{1+\frac{d}{2}}\right)}}_{\leq \delta}
\end{aligned}
$$




$$
\leq c \delta\left(1-e^{-c_{2} T}\right)\left\|u^{(n+1)}\right\|_{L^{\infty}\left(0, T, B_{2,1}^{1+\frac{d}{2}-2 \alpha}\right)} .
$$

The terms with $J_{2}$ and $J_{3}$ can be similarly estimated and obey the same bound.

$$
\begin{aligned}
\int_{0}^{T} & \sum_{j \geq-1} 2^{\left(1+\frac{d}{2}\right) j} \int_{0}^{s} e^{-c_{0} 2^{2 \alpha j}(s-\tau)} J_{2} d \tau d s \\
& =c \int_{0}^{T} \sum_{j \geq-1} 2^{\left(1+\frac{d}{2}\right) j} \int_{0}^{s} e^{-c_{0} 2^{2 \alpha j}(s-\tau)}\left\|\Delta_{j} u^{(n)}\right\|_{L^{2}} \sum_{m \leq j} 2^{\left(1+\frac{d}{2}\right) m}\left\|\Delta_{m} u^{(n+1)}\right\|_{L^{2}} d \tau d s \\
& \leq c \sum_{j \geq-1} 2^{\left(1+\frac{d}{2}\right) j} \int_{0}^{T}\left\|\Delta_{j} u^{(n)}\right\|_{L^{2}} \sum_{m \leq j} 2^{\left(1+\frac{d}{2}\right) m}\left\|\Delta_{m} u^{(n+1)}\right\|_{L^{2}} d \tau \int_{0}^{T} e^{-c_{0} 2^{2 \alpha j} s} d s .
\end{aligned}
$$

Owing to (3.8) and the above inequality,

$$
\begin{aligned}
& \int_{0}^{T} \sum_{j \geq-1} 2^{\left(1+\frac{d}{2}\right) j} \int_{0}^{s} e^{-c_{0} 2^{2 \alpha j}(s-\tau)} J_{2} d \tau d s \\
& \leq c\left(1-e^{-c_{2} T}\right) \int_{0}^{T} \sum_{j \geq-1} 2^{\left(1+\frac{d}{2}-2 \alpha\right) j}\left\|\Delta_{j} u^{(n)}\right\|_{L^{2}} \sum_{m \leq j-1} 2^{\left(1+\frac{d}{2}\right) m}\left\|\Delta_{m} u^{(n+1)}\right\|_{L^{2}} d \tau \\
& \leq c\left(1-e^{-c_{2} T}\right) \int_{0}^{T} \sum_{j \geq-1} 2^{\left(1+\frac{d}{2}-2 \alpha\right) j}\left\|\Delta_{j} u^{(n+1)}\right\|_{L^{2}} \sum_{j \geq-1} 2^{\left(1+\frac{d}{2}\right) j}\left\|\Delta_{j} u^{(n)}\right\|_{L^{2}} d \tau \\
& \leq c\left(1-e^{-c_{2} T}\right)\left\|u^{(n+1)}\right\|_{L^{\infty}\left(0, T, B_{2,1}^{1+\frac{d}{2}-2 \alpha}\right)} \underbrace{\left\|u^{(n)}\right\|_{L^{1}\left(0, T, B_{2,1}^{1+\frac{d}{2}}\right)}}_{\leq \delta} \\
& \leq c\left(1-e^{-c_{2} T}\right) \delta\left\|u^{(n+1)}\right\|_{L^{\infty}\left(0, T, B_{2,1}^{1+\frac{d}{2}-2 \alpha}\right)} .
\end{aligned}
$$

Similarly

$$
\begin{aligned}
\int_{0}^{T} \sum_{j \geq-1} 2^{\left(1+\frac{d}{2}\right) j} & \int_{0}^{s} e^{-c_{0} 2^{2 \alpha j}(s-\tau)} J_{3} d \tau d s \\
& =c \int_{0}^{T} \sum_{j \geq-1} 2^{\left(2+\frac{d}{2}\right) j} \int_{0}^{s} e^{-c_{0} 2^{2 \alpha j}(s-\tau)} \sum_{k \geq j-1} 2^{\frac{d}{2} k}\left\|\Delta_{k} u^{(n)}\right\|_{L^{2}}\left\|\widetilde{\Delta}_{k} u^{(n+1)}\right\|_{L^{2}} d \tau d s \\
& \leq c \sum_{j \geq-1} 2^{\left(2+\frac{d}{2}\right) j} \int_{0}^{T} \sum_{k \geq j-1} 2^{\frac{d}{2} k}\left\|\Delta_{k} u^{(n)}\right\|_{L^{2}}\left\|\widetilde{\Delta}_{k} u^{(n+1)}\right\|_{L^{2}} d \tau \int_{0}^{T} e^{-c_{0} 2^{2 \alpha j} s} d s
\end{aligned}
$$

Then, due to (3.8),

$$
\int_{0}^{T} \sum_{j \geq-1} 2^{\left(1+\frac{d}{2}\right) j} \int_{0}^{s} e^{-c_{0} 2^{2 \alpha j}(s-\tau)} J_{3} d \tau d s
$$




$$
\begin{aligned}
& \leq c\left(1-e^{-c_{2} T}\right) \int_{0}^{T} \sum_{j \geq-1} 2^{\left(2+\frac{d}{2}-2 \alpha\right) j} \sum_{k \geq j-1} 2^{\frac{d}{2} k}\left\|\Delta_{k} u^{(n)}\right\|_{L^{2}}\left\|\widetilde{\Delta}_{k} u^{(n+1)}\right\|_{L^{2}} d \tau \\
& \leq c\left(1-e^{-c_{2} T}\right) \int_{0}^{T} \sum_{j \geq-1} 2^{\left(2+\frac{d}{2}-2 \alpha\right) j}\left\|\widetilde{\Delta}_{j} u^{(n+1)}\right\|_{L^{2}} \sum_{j \geq-1} 2^{\left(\frac{d}{2}\right) j}\left\|\Delta_{j} u^{(n)}\right\|_{L^{2}} d \tau \\
& \leq c\left(1-e^{-c_{2} T}\right) \int_{0}^{T} \sum_{j \geq-1} 2^{\left(1+\frac{d}{2}-2 \alpha\right) j}\left\|\widetilde{\Delta}_{j} u^{(n+1)}\right\|_{L^{2}} \sum_{j \geq-1} 2^{\left(1+\frac{d}{2}\right) j}\left\|\Delta_{j} u^{(n)}\right\|_{L^{2}} d \tau \\
& \leq c\left(1-e^{-c_{2} T}\right)\left\|u^{(n+1)}\right\|_{L^{\infty}\left(0, T, B_{2,1}^{1+\frac{d}{2}-2 \alpha}\right)} \underbrace{\left.\left\|u^{(n)}\right\|_{L^{1}\left(0, T, B_{2,1}\right.}^{1+\frac{d}{2}}\right)}_{\leq \delta} \\
& \leq c\left(1-e^{-c_{2} T}\right) \delta\left\|u^{(n+1)}\right\|_{L^{\infty}\left(0, T, B_{2,1}^{1+\frac{d}{2}-2 \alpha}\right)} \cdot
\end{aligned}
$$

Now, for the term with $J_{4}$ we write

$$
\begin{aligned}
& \int_{0}^{T} \sum_{j \geq-1} 2^{\left(1+\frac{d}{2}\right) j} \int_{0}^{s} e^{-c_{0} 2^{2 \alpha j}(s-\tau)} J_{4} d \tau d s=c \int_{0}^{T} \sum_{j \geq-1} 2^{\left(2+\frac{d}{2}\right) j} \int_{0}^{s} e^{-c_{0} 2^{2 \alpha j}(s-\tau)}\left\|\Delta_{j} w^{(n)}\right\|_{L^{2}} d \tau d s \\
& \leq c \sum_{j \geq-1} 2^{\left(2+\frac{d}{2}\right) j} \int_{0}^{T}\left\|\Delta_{j} w^{(n)}\right\|_{L^{2}} d \tau \int_{0}^{T} e^{-c_{0} 2^{2 \alpha j} s} d s \\
& \leq c\left(1-e^{-c_{2} T}\right) \int_{0}^{T} \sum_{j \geq-1} 2^{\left(2+\frac{d}{2}-2 \alpha\right)}\left\|\Delta_{j} w^{(n)}\right\|_{L^{2}} d \tau \\
& \underset{\text { since } \alpha \geq \frac{1}{2}}{\leq} c\left(1-e^{-c_{2} T}\right) \underbrace{\int_{0}^{T} \sum_{j \geq-1} 2^{\left(1+\frac{d}{2}\right) j}\left\|\Delta_{j} w^{(n)}\right\|_{L^{2}} d \tau}_{=\left\|w^{(n)}\right\|_{L^{1}\left(0, T, B_{2,1}^{1+\frac{d}{2}}\right)} \leq \delta} \\
& \leq c\left(1-e^{-c_{2} T}\right) \delta .
\end{aligned}
$$

Collecting the estimates above leads to

$$
\begin{aligned}
\left\|u^{(n+1)}\right\|_{L^{1}\left(0, T, B_{2,1}^{1+\frac{d}{2}}\right)} & \leq \frac{\delta}{4}+c \delta\left(1-e^{-c_{2} T}\right) \| u_{L^{\infty}\left(0, T, B_{2,1}^{1+\frac{d}{2}}\right)}^{(n+1)}+c\left(1-e^{-c_{2} T}\right) \delta \\
& \leq \frac{\delta}{4}+c \delta\left(1-e^{-c_{2} T}\right) M+c\left(1-e^{-c_{2} T}\right) \delta .
\end{aligned}
$$

Choosing T sufficiently small such that $c\left(1-e^{-c_{2} T}\right) \leq \min \left(\frac{1}{4 M}, \frac{1}{2}\right)$ we get

$$
\left\|u^{(n+1)}\right\|_{L^{1}\left(0, T, B_{2,1}^{1+\frac{d}{2}}\right)} \leq \frac{\delta}{4}+\frac{\delta}{4}+\frac{\delta}{2}=\delta .
$$

3.1.3 The estimate of $w^{(n+1)}$ in $L^{\infty}\left(0, T, B_{2,1}^{1+\frac{d}{2}-2 \beta}\left(\mathbb{R}^{d}\right)\right)$. We apply $\Delta_{j}$ to the third equation in (3.1) and then dotting with $\Delta_{j} w^{(n+1)}$, we obtain

$$
\frac{1}{2} \frac{d}{d t}\left\|\Delta_{j} w^{(n+1)}\right\|_{L^{2}}^{2}+\left(c_{1} 2^{2 \beta j}+4 k\right)\left\|\Delta_{j} w^{(n+1)}\right\|_{L^{2}}^{2}
$$




$$
\begin{aligned}
& \leq-2 k \int \Delta_{j}\left(\nabla \times u^{(n)}\right) \Delta_{j} w^{(n+1)} d x \\
& -\int \Delta_{j}\left(u^{(n)} \cdot \nabla w^{(n+1)}\right) \Delta_{j} w^{(n+1)} d x \\
& =B_{1}+B_{2},
\end{aligned}
$$

where

$$
\begin{aligned}
& B_{1}=-2 k \int \Delta_{j}\left(\nabla \times u^{(n)}\right) \Delta_{j} w^{(n+1)} d x, \\
& B_{2}=-\int \Delta_{j}\left(u^{(n)} \cdot \nabla w^{(n+1)}\right) \Delta_{j} w^{(n+1)} d x .
\end{aligned}
$$

By Hölder's inequality and Bernstein's inequality,

$$
\begin{aligned}
\left|B_{1}\right| & =\left|-2 k \int \Delta_{j}\left(\nabla \times u^{(n)}\right) \Delta_{j} w^{(n+1)} d x\right| \\
& \leq 2 k\left\|\Delta_{j}\left(\nabla \times u^{(n)}\right)\right\|_{L^{2}}\left\|\Delta_{j} w^{(n+1)}\right\|_{L^{2}} \\
& \leq c 2^{j}\left\|\Delta_{j} u^{(n)}\right\|_{L^{2}}\left\|\Delta_{j} w^{(n+1)}\right\|_{L^{2}} .
\end{aligned}
$$

By Lemma 2.6,

$$
\begin{aligned}
\left|B_{2}\right| & =\left|-\int \Delta_{j}\left(u^{(n)} \cdot \nabla w^{(n+1)}\right) \Delta_{j} w^{(n+1)} d x\right| \\
& \leq c\left\|\Delta_{j} w^{(n+1)}\right\|_{L^{2}}^{2} \sum_{m \leq j-1} 2^{\left(1+\frac{d}{2}\right) m}\left\|\Delta_{m} u^{(n)}\right\|_{L^{2}} \\
& +c\left\|\Delta_{j} w^{(n+1)}\right\|_{L^{2}}\left\|\Delta_{j} u^{(n)}\right\|_{L^{2}} \sum_{m \leq j} 2^{\left(1+\frac{d}{2}\right) m}\left\|_{m} w^{(n+1)}\right\|_{L^{2}} \\
& +c\left\|\Delta_{j} w^{(n+1)}\right\|_{L^{2}} 2^{j} \sum_{k \geq j-1} 2^{\frac{d}{2} k}\left\|\widetilde{\Delta}_{k} w^{(n+1)}\right\|_{L^{2}}\left\|\Delta_{k} u^{(n)}\right\|_{L^{2}} .
\end{aligned}
$$

Inserting the estimates above in (3.9) and eliminating $\left\|\Delta_{j} w^{(n+1)}\right\|_{L^{2}}$ from both sides of the inequality, we obtain

$$
\frac{d}{d t}\left\|\Delta_{j} w^{(n+1)}\right\|_{L^{2}}+\left(c_{1} 2^{2 \beta j}+4 k\right)\left\|\Delta_{j} w^{(n+1)}\right\|_{L^{2}} \leq K_{1}+K_{2}+K_{3}+K_{4},
$$

where

$$
\begin{aligned}
& \left.K_{1}=c 2^{j} \| \Delta_{j} u^{(n)}\right) \|_{L^{2}}, \\
& K_{2}=c\left\|\Delta_{j} w^{(n+1)}\right\|_{L^{2}} \sum_{m \leq j-1} 2^{\left(1+\frac{d}{2}\right) m}\left\|\Delta_{m} u^{(n)}\right\|_{L^{2}}, \\
& K_{3}=c\left\|\Delta_{j} u^{(n)}\right\|_{L^{2}} \sum_{m \leq j} 2^{\left(1+\frac{d}{2}\right) m}\left\|\Delta_{m} w^{(n+1)}\right\|_{L^{2}},
\end{aligned}
$$




$$
K_{4}=c \sum_{k \geq j-1} 2^{j} 2^{\frac{d}{2} k}\left\|\widetilde{\Delta}_{k} w^{(n+1)}\right\|_{L^{2}}\left\|\Delta_{k} u^{(n)}\right\|_{L^{2}}
$$

Integrating (3.10) in time yields, for any $t \leq T$,

$$
\left\|\Delta_{j} w^{(n+1)}\right\|_{L^{2}} \leq e^{-\left(c_{1} 2^{2 \beta j}\right) t}\left\|\Delta_{j} w_{0}^{(n+1)}\right\|_{L^{2}}+\int_{0}^{t} e^{-\left(c_{1} 2^{2 \beta j}\right)(t-\tau)}\left(K_{1}+\cdots+K_{4}\right) d \tau .
$$

Multiplying (3.11) by $2^{\left(1+\frac{d}{2}-2 \beta\right) j}$ and summing over $j$, we have

$$
\left\|w^{(n+1)}\right\|_{B_{2,1}^{1+\frac{d}{2}-2 \beta}} \leq\left\|w_{0}^{(n+1)}\right\|_{B_{2,1}^{1+\frac{d}{2}-2 \beta}}+\sum_{j \geq-1} \int_{0}^{t} e^{-\left(c_{1} 2^{2 \beta j}\right)(t-\tau)} 2^{\left(1+\frac{d}{2}-2 \beta\right) j}\left(K_{1}+\cdots+K_{4}\right) d \tau .
$$

The terms containing $K_{1}$ through $K_{4}$ on the right hand side of (3.12) can be bounded suitably as follows. We start with the term with $K_{1}$,

$$
\begin{aligned}
\sum_{j \geq-1} \int_{0}^{t} 2^{\left(1+\frac{d}{2}-2 \beta\right) j} K_{1} d \tau & =\int_{0}^{t} \sum_{j \geq-1} c 2^{\left(2+\frac{d}{2}-2 \beta\right) j}\left\|\Delta_{j} u^{(n)}(\tau)\right\|_{L^{2}} d \tau \\
\leq & c \\
\text { since } \beta \geq \frac{1}{2} & \underbrace{\int_{0}^{t} \sum_{j \geq-1} 2^{\left(1+\frac{d}{2}\right) j}\left\|\Delta_{j} u^{(n)}(\tau)\right\|_{L^{2}} d \tau}_{\left\|u^{(n)}\right\|_{L^{1}\left(0, T, B_{2,1}^{1+\frac{d}{2}}\right.} \leq \delta} \leq c \delta .
\end{aligned}
$$

Similarly the term with $K_{2}$ is bounded by

$$
\begin{aligned}
\sum_{j \geq-1} 2^{\left(1+\frac{d}{2}-2 \beta\right) j} \int_{0}^{t} K_{2} d \tau & =c \int_{0}^{t} \sum_{j \geq-1} 2^{\left(1+\frac{d}{2}-2 \beta\right) j}\left\|\Delta_{j} w^{(n+1)}\right\|_{L^{2}} \sum_{m \leq j-1} 2^{\left(1+\frac{d}{2}\right) m}\left\|\Delta_{m} u^{(n)}\right\|_{L^{2}} d \tau \\
& \leq c\left\|w^{(n+1)}\right\|_{L^{\infty}\left(0, T, B_{2,1}^{1+\frac{d}{2}-2 \beta}\right)}\left\|u^{(n)}\right\|_{L^{1}\left(0, T, B_{2,1}^{1+\frac{d}{2}}\right)} \\
& \leq c \delta\left\|w^{(n+1)}\right\|_{L^{\infty}\left(0, T, B_{2,1}^{1+\frac{d}{2}-2 \beta}\right)} .
\end{aligned}
$$

The terms related to $K_{3}$ and $K_{4}$ obey also the same bound,

$$
\begin{aligned}
\sum_{j \geq-1} 2^{\left(1+\frac{d}{2}-2 \beta\right) j} \int_{0}^{t} K_{3} d \tau & =c \int_{0}^{t} \sum_{j \geq-1} 2^{\left(1+\frac{d}{2}-2 \beta\right) j}\left\|\Delta_{j} u^{(n)}\right\|_{L^{2}} \sum_{m \leq j} 2^{\left(1+\frac{d}{2}\right) m}\left\|\Delta_{m} w^{(n+1)}\right\|_{L^{2}} d \tau \\
& \leq c\left\|w^{(n+1)}\right\|_{L^{\infty}\left(0, T, B_{2,1}^{1+\frac{d}{2}-2 \beta}\right)}\left\|u^{(n)}\right\|_{L^{1}\left(0, T, B_{2,1}^{1+\frac{d}{2}}\right)} \\
& \leq c \delta\left\|w^{(n+1)}\right\|_{L^{\infty}\left(0, T, B_{2,1}^{1+\frac{d}{2}-2 \beta}\right)} .
\end{aligned}
$$

For the term with $K_{4}$ we write

$$
\sum_{j \geq-1} 2^{\left(1+\frac{d}{2}-2 \beta\right) j} \int_{0}^{t} K_{4} d \tau=c \int_{0}^{t} \sum_{j \geq-1} \sum_{k \geq j-1} 2^{\left(2+\frac{d}{2}-2 \beta\right) j} 2^{\frac{d}{2} k}\left\|\widetilde{\Delta}_{k} w^{(n+1)}\right\|_{L^{2}}\left\|\Delta_{k} u^{(n)}\right\|_{L^{2}} d \tau
$$




$$
\begin{aligned}
& \leq c \int_{0}^{t} \sum_{j \geq-1} \sum_{j \geq-1} 2^{\left(2+\frac{d}{2}-2 \beta\right) j} 2^{\frac{d}{2} j}\left\|\widetilde{\Delta}_{j} w^{(n+1)}\right\|_{L^{2}}\left\|\Delta_{j} u^{(n)}\right\|_{L^{2}} d \tau \\
& =c \int_{0}^{t} \sum_{j \geq-1} \sum_{j \geq-1} 2^{\left(1+\frac{d}{2}-2 \beta\right) j} 2^{\left(1+\frac{d}{2}\right) j}\left\|\widetilde{\Delta}_{j} w^{(n+1)}\right\|_{L^{2}}\left\|\Delta_{j} u^{(n)}\right\|_{L^{2}} d \tau \\
& \leq c\left\|w^{(n+1)}\right\|_{L^{\infty}\left(0, T, B_{2,1}^{1+\frac{d}{2}-2 \beta}\right)}\left\|u^{(n)}\right\|_{L^{1}\left(0, T, B_{2,1}^{1+\frac{d}{2}}\right)} \\
& \leq c \delta\left\|w^{(n+1)}\right\|_{L^{\infty}\left(0, T, B_{2,1}^{1+\frac{d}{2}-2 \beta}\right)} .
\end{aligned}
$$

Collecting the estimates and inserting them in (3.12), we obtain for any $t \leq T$

$$
\left\|w^{(n+1)}(t)\right\|_{B_{2,1}^{1+\frac{d}{2}-2 \beta}} \leq\left\|w_{0}^{(n+1)}\right\|_{B_{2,1}^{1+\frac{d}{2}-2 \beta}}+c \delta+c \delta\left\|w^{(n+1)}\right\|_{L^{\infty}\left(0, T, B_{2,1}^{1+\frac{d}{2}-2 \beta}\right)} .
$$

Choosing $c \delta \leq \min \left(\frac{1}{4}, \frac{M}{4}\right)$, we get

$$
\left\|w^{(n+1)}(t)\right\|_{L^{\infty}\left(0, T, B_{2,1}^{1+\frac{d}{2}-2 \beta}\right)} \leq \frac{M}{2}+\frac{M}{4}+\frac{1}{4}\left\|w^{(n+1)}(t)\right\|_{L^{\infty}\left(0, T, B_{2,1}^{1+\frac{d}{2}-2 \beta}\right)},
$$

which implies

$$
\left\|w^{(n+1)}(t)\right\|_{L^{\infty}\left(0, T, B_{2,1}^{1+\frac{d}{2}-2 \beta}\right)} \leq M
$$

3.1.4 The estimate of $\left\|w^{(n+1)}(t)\right\|_{L^{1}\left(0, T, B_{2,1}^{1+\frac{d}{2}}\right)}$. We recall $(3.11)$

$$
\left\|\Delta_{j} w^{(n+1)}\right\|_{L^{2}} \leq e^{-\left(c_{1} 2^{2 \beta j}\right) t}\left\|\Delta_{j} w_{0}^{(n+1)}\right\|_{L^{2}}+\int_{0}^{t} e^{-c_{1} 2^{2 \beta j}(t-\tau)}\left(K_{1}+\cdots+K_{4}\right) d \tau
$$

We multiply by $2^{\left(1+\frac{d}{2}\right) j}$, sum over $j$ and integrate in time to get

$$
\begin{aligned}
\left\|w^{(n+1)}\right\|_{L^{1}\left(0, T, B_{2,1}^{1+\frac{d}{2}}\right)} & \leq \int_{0}^{T} \sum_{j \geq-1} 2^{\left(1+\frac{d}{2}\right) j} e^{-c_{1} 2^{2 \beta j} t}\left\|\Delta_{j} w_{0}^{(n+1)}\right\|_{L^{2}} d t \\
& +\int_{0}^{T} \sum_{j \geq-1} 2^{\left(1+\frac{d}{2}\right) j} \int_{0}^{s} e^{-c_{1} 2^{2 \beta j}(s-\tau)}\left(K_{1}+\cdots+K_{4}\right) d \tau d s .
\end{aligned}
$$

Clearly

$$
\int_{0}^{T} \sum_{j \geq-1} 2^{\left(1+\frac{d}{2}\right) j} e^{-c_{1} 2^{2 \beta j} t}\left\|\Delta_{j} w_{0}^{(n+1)}\right\|_{L^{2}} d t=c \sum_{j \geq-1} 2^{\left(1+\frac{d}{2}-2 \beta\right) j}\left(1-e^{-c_{1} 2^{2 \beta j} T}\right)\left\|\Delta_{j} w_{0}^{(n+1)}\right\|_{L^{2}} .
$$

Since $w_{0} \in B_{2,1}^{1+\frac{d}{2}-2 \beta}$, we have by the Dominated Convergence Theorem,

$$
\lim _{T \rightarrow 0} \sum_{j \geq-1} 2^{\left(1+\frac{d}{2}-2 \beta\right) j}\left(1-e^{-c_{1} 2^{2 \beta j} T}\right)\left\|\Delta_{j} w_{0}^{(n+1)}\right\|_{L^{2}}=0 .
$$


Therefore, we can choose $T$ sufficiently small

$$
\int_{0}^{T} \sum_{j \geq-1} 2^{\left(1+\frac{d}{2}\right) j} e^{-c_{1} 2^{2 \beta j} t}\left\|\Delta_{j} w_{0}^{(n+1)}\right\|_{L^{2}} d t \leq \frac{\delta}{2} .
$$

Applying Young's inequality for the time convolution, the term with $K_{1}$ is bounded by

$$
\begin{aligned}
\int_{0}^{T} \sum_{j \geq-1} 2^{\left(1+\frac{d}{2}\right) j} \int_{0}^{s} e^{-c_{1} 2^{2 \beta j}(s-\tau)} K_{1} d \tau d s & \leq c \int_{0}^{T} \sum_{j \geq-1} 2^{\left(2+\frac{d}{2}\right) j} \int_{0}^{s} e^{-c_{1} 2^{2 \beta j}(s-\tau)}\left\|\Delta_{j} u^{(n)}\right\|_{L^{2}} d \tau d s \\
& \leq c \sum_{j \geq-1} 2^{\left(2+\frac{d}{2}\right) j} \int_{0}^{T}\left\|\Delta_{j} u^{(n)}\right\|_{L^{2}} d \tau \cdot \int_{0}^{T} e^{-c_{1} 2^{2 \beta j} s} d s .
\end{aligned}
$$

Using the fact that there exists $c_{3}>0$ satisfying for all $j \geq 0$,

$$
\int_{0}^{T} e^{-c_{1} 2^{2 \beta j}} d s \leq c 2^{-2 \beta j}\left(1-e^{-c_{3} T}\right),
$$

we get

$$
\begin{aligned}
\int_{0}^{T} \sum_{j \geq-1} 2^{\left(1+\frac{d}{2}\right) j} \int_{0}^{s} e^{-c_{1} 2^{2 \beta j}(s-\tau)} K_{1} d \tau d s & \leq c\left(1-e^{-c_{3} T}\right) \int_{0}^{T} \sum_{j \geq-1} 2^{\left(2+\frac{d}{2}-2 \beta\right) j}\left\|\Delta_{j} u^{(n)}(\tau)\right\|_{L^{2}} d \tau \\
& \leq c\left(1-e^{-c_{3} T}\right) \underbrace{\int_{0}^{T} \sum_{j \geq-1}}_{\left.=\left\|u^{(n+1)}\right\|_{L^{1}\left(0, T, B_{2,1}\right.}^{1+\frac{d}{2}}\right)} 2^{\left(1+\frac{d}{2}\right) j}\left\|\Delta_{j} u^{(n)}(\tau)\right\|_{L^{2}} d \tau \\
& \leq c\left(1-e^{-c_{3} T}\right) \delta .
\end{aligned}
$$

Similarly by applying Young's inequality for the time convolution, the term with $K_{2}$ is bounded by

$$
\begin{aligned}
& \int_{0}^{T} \sum_{j \geq-1} 2^{\left(1+\frac{d}{2}\right) j} \int_{0}^{s} e^{-c_{1} 2^{2 \beta j}(s-\tau)} K_{2} d \tau d s \\
& =c \int_{0}^{T} \sum_{j \geq-1} 2^{\left(1+\frac{d}{2}\right) j} \int_{0}^{s} e^{-c_{1} 2^{2 \beta j}(s-\tau)} c\left\|\Delta_{j} w^{(n+1)}\right\|_{L^{2}} \sum_{m \leq j-1} 2^{\left(1+\frac{d}{2}\right) m}\left\|\Delta_{m} u^{(n)}(\tau)\right\|_{L^{2}} d \tau d s \\
& \leq c \sum_{j \geq-1} 2^{\left(1+\frac{d}{2}\right) j} \int_{0}^{T}\left\|\Delta_{j} w^{(n+1)}\right\|_{L^{2}} \sum_{m \leq j-1} 2^{\left(1+\frac{d}{2}\right) m}\left\|\Delta_{m} u^{(n)}(\tau)\right\|_{L^{2}} d \tau\left(\int_{0}^{T} e^{-c_{1} 2^{2 \beta j} s} d s\right) \\
& \leq c\left(1-e^{-c_{3} T}\right) \int_{0}^{T} \sum_{j \geq-1} 2^{\left(1+\frac{d}{2}-2 \beta\right) j}\left\|\Delta_{j} w^{(n+1)}(\tau)\right\|_{L^{2}} \sum_{m \leq j-1} 2^{\left(1+\frac{d}{2}\right) m}\left\|\Delta_{m} u^{(n)}(\tau)\right\|_{L^{2}} d \tau \\
& \leq c\left(1-e^{-c_{3} T}\right) \underbrace{\left\|w^{(n+1)}\right\|_{L^{\infty}\left(0, T, B_{2,1}^{1+\frac{d}{2}-2 \beta}\right)}^{\left\|u^{(n)}\right\|_{L^{1}\left(0, T, B_{2,1}^{1+\frac{d}{2}}\right)}}}_{\leq M} \underbrace{}_{\leq \delta}
\end{aligned}
$$




$$
\leq c\left(1-e^{-c_{3} T}\right) \delta M
$$

The terms involving $K_{3}$ and $K_{4}$ obey also the same bound,

$$
\begin{aligned}
& \int_{0}^{T} \sum_{j \geq-1} 2^{\left(1+\frac{d}{2}\right) j} \int_{0}^{s} e^{-c_{1} 2^{2 \beta j}(s-\tau)} K_{3} d \tau d s \\
& \quad=c \int_{0}^{T} \sum_{j \geq-1} 2^{\left(1+\frac{d}{2}\right) j} \int_{0}^{s} e^{-c_{1} 2^{2 \beta j}(s-\tau)} c\left\|\Delta_{j} u^{(n)}(\tau)\right\|_{L^{2}}\left(\sum_{m \leq j} 2^{\left(1+\frac{d}{2}\right) m}\left\|\Delta_{m} w^{(n+1)}(\tau)\right\|_{L^{2}}\right) d \tau d s \\
& \leq c \sum_{j \geq-1} 2^{\left(1+\frac{d}{2}\right) j} \int_{0}^{T}\left\|\Delta_{j} u^{(n)}(\tau)\right\|_{L^{2}} \sum_{m \leq j} 2^{\left(1+\frac{d}{2}\right) m}\left\|\Delta_{m} w^{(n+1)}(\tau)\right\|_{L^{2}} d \tau\left(\int_{0}^{T} e^{-c_{1} 2^{2 \beta j} s} d s\right)
\end{aligned}
$$

Then, owing to (3.14)

$$
\begin{aligned}
\int_{0}^{T} \sum_{j \geq-1} & 2^{\left(1+\frac{d}{2}\right) j} \int_{0}^{s} e^{-c_{1} 2^{2 \beta j}(s-\tau)} K_{3} d \tau d s \\
& \leq c\left(1-e^{-c_{3} T}\right) \int_{0}^{T} \sum_{j \geq-1} 2^{\left(1+\frac{d}{2}-2 \beta\right) j}\left\|\Delta_{j} u^{(n)}(\tau)\right\|_{L^{2}} \sum_{m \leq j} 2^{\left(1+\frac{d}{2}\right) m}\left\|\Delta_{m} w^{(n+1)}(\tau)\right\|_{L^{2}} d \tau \\
& \leq c\left(1-e^{-c_{3} T}\right) \int_{0}^{T} \sum_{j \geq-1} 2^{\left(1+\frac{d}{2}-2 \beta\right) j}\left\|\Delta_{j} u^{(n)}(\tau)\right\|_{L^{2}} \sum_{j \geq-1} 2^{\left(1+\frac{d}{2}\right) j}\left\|\Delta_{j} w^{(n+1)}(\tau)\right\|_{L^{2}} d \tau \\
& =c\left(1-e^{-c_{3} T}\right) \int_{0}^{T} \sum_{j \geq-1} 2^{\left(1+\frac{d}{2}\right) j}\left\|\Delta_{j} u^{(n)}(\tau)\right\|_{L^{2}} \sum_{j \geq-1} 2^{\left(1+\frac{d}{2}-2 \beta\right) j}\left\|\Delta_{j} w^{(n+1)}(\tau)\right\|_{L^{2}} d \tau \\
& \leq c\left(1-e^{-c_{3} T}\right) \underbrace{\underbrace{}_{L^{\infty}\left(0, T, B_{2,1}^{1+\frac{d}{2}-2 \beta}\right)}}_{\leq w^{(n+1)} \|} \underbrace{\left\|u^{(n)}\right\| \|_{L^{1}\left(0, T, B_{2,1}^{1+\frac{d}{2}}\right)}}_{\leq \delta} \\
& \leq c\left(1-e^{-c_{3} T}\right) \delta M .
\end{aligned}
$$

The term containing $K_{4}$ is bounded by

$$
\begin{aligned}
& \int_{0}^{T} \sum_{j \geq-1} 2^{\left(1+\frac{d}{2}\right) j} \int_{0}^{s} e^{-c_{1} 2^{2 \beta j}(s-\tau)} K_{4} d \tau d s \\
& \quad=c \int_{0}^{T} \sum_{j \geq-1} 2^{\left(1+\frac{d}{2}\right) j} \int_{0}^{s} e^{-c_{1} 2^{2 \beta j}(s-\tau)} 2^{j} 2^{\frac{d}{2} k}\left\|\widetilde{\Delta}_{k} w^{(n+1)}\right\|_{L^{2}}\left\|\Delta_{k} u^{(n)}\right\|_{L^{2}} d \tau d s \\
& \quad \leq c \sum_{j \geq-1} 2^{\left(1+\frac{d}{2}\right) j} \int_{0}^{T} \sum_{k \geq j-1} 2^{j} 2^{\frac{d}{2} k}\left\|\widetilde{\Delta}_{k} w^{(n+1)}\right\|_{L^{2}}\left\|\Delta_{k} u^{(n)}\right\|_{L^{2}} d \tau\left(\int_{0}^{T} e^{-c_{1} 2^{2 \beta j} s} d s\right) .
\end{aligned}
$$

Hence, due to (3.14)

$$
\int_{0}^{T} \sum_{j \geq-1} 2^{\left(1+\frac{d}{2}\right) j} \int_{0}^{s} e^{-c_{1} 2^{2 \beta j}(s-\tau)} K_{4} d \tau d s
$$




$$
\begin{aligned}
& \leq c\left(1-e^{-c_{3} T}\right) \int_{0}^{T} \sum_{j \geq-1} 2^{\left(1+\frac{d}{2}-2 \beta\right) j} 2^{j} \sum_{k \geq j-1} 2^{\frac{d}{2} k}\left\|\widetilde{\Delta}_{k} w^{(n+1)}\right\|_{L^{2}}\left\|\Delta_{k} u^{(n)}\right\|_{L^{2}} d \tau \\
& =c\left(1-e^{-c_{3} T}\right) \int_{0}^{T} \sum_{j \geq-1} \sum_{k \geq j-1} 2^{\left(1+\frac{d}{2}-2 \beta\right)(j-k)} 2^{\left(1+\frac{d}{2}\right) k}\left\|\Delta_{k} u^{(n)}\right\|_{L^{2}} 2^{\left(1+\frac{d}{2}-2 \beta\right) k}\left\|\widetilde{\Delta}_{k} w^{(n+1)}\right\|_{L^{2}} d \tau \\
& =c\left(1-e^{-c_{3} T}\right) \int_{0}^{\int_{0}^{T}\left\|u^{(n)}\right\|_{B_{2,1}^{1+\frac{d}{2}}}\left\|w^{(n+1)}(\tau)\right\|_{B_{2,1}^{1+\frac{d}{2}-2 \beta}} d \tau} \underbrace{\leq c\left(1-e^{-c_{3} T}\right) \underbrace{}_{u^{(n)} \|} \underbrace{}_{L^{1}\left(0, T, B_{2,1}^{1+\frac{d}{2}}\right)} \underbrace{\left\|w^{(n+1)}\right\|_{L_{2,1}}}_{\leq M}}_{\leq \delta} \\
& \leq c\left(1-e^{-c_{3} T}\right) \delta M .
\end{aligned}
$$

Collecting the estimates above and inserting them in (3.13), we obtain

$$
\left\|w^{(n+1)}\right\|_{L^{1}\left(0, T, B_{2,1}^{1+\frac{d}{2}}\right)} \leq \frac{\delta}{2}+c\left(1-e^{-c_{3} T}\right) \delta+c\left(1-e^{-c_{3} T}\right) \delta M .
$$

Choosing T sufficiently small such that $c\left(1-e^{-c_{3} T}\right) \leq \min \left(\frac{1}{4 M}, \frac{1}{4}\right)$, we get

$$
\left\|w^{(n+1)}\right\|_{L^{1}\left(0, T, B_{2,1}^{1+\frac{d}{2}}\right)} \leq \frac{\delta}{2}+\frac{\delta}{4}+\frac{\delta}{4}=\delta .
$$

These uniform bounds allow us to extract a weakly convergent subsequence. That is, there is $(u, w) \in Y$ such that subsequence of $\left(u^{n}, w^{n}\right)$ (still denoted by $\left(u^{n}, w^{n}\right)$ ) satisfies

$$
\begin{aligned}
& u^{n} \stackrel{*}{\rightarrow} u \quad \text { in } \quad L^{\infty}\left(0, T, B_{2,1}^{1+\frac{d}{2}-2 \alpha}\right), \\
& w^{n} \stackrel{*}{\rightarrow} w \quad \text { in } \quad L^{\infty}\left(0, T, B_{2,1}^{1+\frac{d}{2}-2 \beta}\right) .
\end{aligned}
$$

In order to show that $(u, w)$ is a weak solution of (1.1) we need to further extract a subsequence which converges strongly to $(u, w)$. We use the Aubin-Lions Lemma. We can show by making use of the equation (3.1) that $\left(\partial_{t} u_{n}, \partial_{t} w_{n}\right)$ is uniformly bounded in

$$
\begin{aligned}
& \partial_{t} u^{n} \in L^{1}\left(0, T, B_{2,1}^{1+\frac{d}{2}-2 \alpha}\right) \cap L^{2}\left(0, T, B_{2,1}^{\frac{d}{2}+1-3 \alpha}\right), \\
& \partial_{t} w^{n} \in L^{1}\left(0, T, B_{2,1}^{1+\frac{d}{2}-2 \beta}\right) \cap L^{2}\left(0, T, B_{2,1}^{\frac{d}{2}+1-3 \beta}\right) .
\end{aligned}
$$

Since we are in this case in the whole space $\mathbb{R}^{d}$, we need to combine Cantor's diagonal process with the Aubin-Lions Lemma to show that a subsequence of a weakly convergent subsequence, still denoted by $\left(u_{n}, w_{n}\right)$, has the following strongly convergent property

$$
\left(u_{n}, w_{n}\right) \longrightarrow(u, w) \text { in } \quad L^{2}\left(0, T, B_{2,1}^{1+\frac{d}{2}-\gamma}(Q)\right),
$$

where $\alpha \leq \gamma \leq 3 \alpha$ and $Q \subset \mathbb{R}^{d}$ is a compact subset. This strong convergence property would allow us to show that $(u, w)$ is indeed a weak solution of (1.1). This completes the proof for the existence part of Theorem 1.1. 


\subsection{Uniqueness of weak solutions}

Proof. Assume that $\left(u^{(1)}, w^{(1)}\right)$ and $\left(u^{(2)}, w^{(2)}\right)$ are two solutions of (1.1) in the regularity class in (1.4) and (1.5). Their difference $(\widetilde{u}, \widetilde{w})$ with

$$
\widetilde{u}=u^{(2)}-u^{(1)} \quad \text { and } \quad \widetilde{w}=w^{(2)}-w^{(1)}
$$

satisfies

$$
\left\{\begin{array}{l}
\partial_{t} \widetilde{u}+(\nu+k)(-\Delta)^{\alpha} \widetilde{u}=-\mathbb{P}\left(u^{(2)} \cdot \nabla \widetilde{u}+\widetilde{u} \cdot \nabla u^{(1)}\right)+2 k \nabla \times \widetilde{w}, \\
\partial_{t} \widetilde{w}+\gamma(-\Delta)^{\beta} \widetilde{w}=-4 k \widetilde{w}-2 k \nabla \times \widetilde{u}-u^{(2)} \cdot \nabla \widetilde{w}-\widetilde{u} \cdot \nabla w^{(1)}, \\
\nabla \cdot \widetilde{u}=0, \\
\widetilde{u}(x, 0)=0, \quad \widetilde{w}(x, 0)=0 .
\end{array}\right.
$$

We estimate the difference $(\widetilde{u}, \widetilde{w})$ in $L^{2}\left(\mathbb{R}^{d}\right)$. Dotting $(3.15)$ by $(\widetilde{u}, \widetilde{w})$ and applying the divergence-free condition, we find

$$
\begin{aligned}
\frac{1}{2} & \frac{d}{d t}\left(\|\widetilde{u}\|_{L^{2}}^{2}+\|\widetilde{w}\|_{L^{2}}^{2}\right)+(\nu+k)\left\|\Lambda^{\alpha} \widetilde{u}\right\|_{L^{2}}^{2}+\gamma\left\|\Lambda^{\beta} \widetilde{w}\right\|_{L^{2}}^{2}+4 k\|\widetilde{w}\|_{L^{2}}^{2} \\
= & -\int u^{(2)} \cdot \nabla \widetilde{u} \cdot \widetilde{u} d x-\int \widetilde{u} \cdot \nabla u^{(1)} \cdot \widetilde{u} d x \\
& -\int u^{(2)} \cdot \nabla \widetilde{w} \cdot \widetilde{w} d x-\int \widetilde{u} \cdot \nabla w^{(1)} \cdot \widetilde{w} d x \\
= & L_{1}+L_{2}+L_{3}+L_{4},
\end{aligned}
$$

where

$$
\begin{aligned}
& L_{1}=-\int u^{(2)} \cdot \nabla \widetilde{u} \cdot \widetilde{u} d x, \\
& L_{2}=-\int \widetilde{u} \cdot \nabla u^{(1)} \cdot \widetilde{u} d x, \\
& L_{3}=-\int u^{(2)} \cdot \nabla \widetilde{w} \cdot \widetilde{w} d x, \\
& L_{4}=-\int \widetilde{u} \cdot \nabla w^{(1)} \cdot \widetilde{w} d x .
\end{aligned}
$$

Due to $\nabla \cdot u^{(2)}=0$, we find $L_{1}=L_{3}=0$ after integration by parts. In fact,

$$
\begin{aligned}
L_{1} & =-\int u^{(2)} \cdot \nabla \widetilde{u} \cdot \widetilde{u} d x \\
& =-\int u^{(2)} \cdot \nabla\left(\frac{1}{2}|\widetilde{u}|^{2}\right) d x
\end{aligned}
$$




$$
\begin{aligned}
& =-\int \nabla \cdot\left(u^{(2)} \frac{1}{2}|\widetilde{u}|^{2}\right) d x \\
& =0 .
\end{aligned}
$$

By Hölder's inequality and Bernstein's inequality,

$$
\begin{aligned}
\left|L_{2}\right| & =\left|-\int \widetilde{u} \cdot \nabla u^{(1)} \cdot \widetilde{u} d x\right| \\
& \leq\left\|\nabla u^{(1)}\right\|_{L^{\infty}}\|\widetilde{u}\|_{L^{2}}^{2} \\
& \leq \sum_{j \geq-1}\left\|\Delta_{j} \nabla u^{(1)}\right\|_{L^{\infty}}\|\widetilde{u}\|_{L^{2}}^{2} \\
& \leq c \underbrace{\sum_{j \geq-1} 2^{d j\left(\frac{1}{2}-\frac{1}{\infty}\right)} 2^{j}\left\|\Delta_{j} u^{(1)}\right\|_{L^{2}}}_{=\left\|u^{(1)}\right\|_{B_{2,1}^{1+\frac{d}{2}}}}\|\widetilde{u}\|_{L^{2}}^{2} \leq c\left\|u^{(1)}\right\|_{B_{2,1}^{1+\frac{d}{2}}}\|\widetilde{u}\|_{L^{2}}^{2} .
\end{aligned}
$$

To bound $L_{4}$, we set

$$
\frac{1}{p}=\frac{1}{2}-\frac{\beta}{d}, \quad \frac{1}{q}=\frac{\beta}{d} \quad\left(\text { or } \frac{d}{q}=\beta\right)
$$

By Hölder's inequality and Bernstein's inequality,

$$
\begin{aligned}
\left|L_{4}\right| & =\left|-\int \widetilde{u} \cdot \nabla w^{(1)} \cdot \widetilde{w} d x\right| \\
& \leq\|\widetilde{u}\|_{L^{2}}\left\|\nabla w^{(1)}\right\|\left\|_{L^{q}}\right\| \widetilde{w} \|_{L^{p}} \\
& \leq \sum_{j \geq-1}\left\|\Delta_{j} \nabla w^{(1)}\right\|_{L^{q}}\|\widetilde{u}\|_{L^{2}}\|\widetilde{w}\|_{L^{p}} \\
& \leq c \sum_{j \geq-1} 2^{j} 2^{d j\left(\frac{1}{2}-\frac{1}{q}\right)}\left\|\Delta_{j} w^{(1)}\right\|_{L^{2}}\|\widetilde{u}\|_{L^{2}}\|\widetilde{w}\|_{L^{p}} \\
& \leq \sum_{j \geq-1} 2^{j+\frac{d j}{2}-2 \beta j}\left\|\Delta_{j} w^{(1)}\right\|_{L^{2}}\|\widetilde{w}\|_{L^{2}}\|\widetilde{u}\|_{L^{p}} \\
& \leq c\left\|w^{(1)}\right\|_{B_{2,1}^{1+\frac{d}{2}-\beta}}\|\widetilde{u}\|_{L^{2}}\left\|\Lambda^{\beta} \widetilde{w}\right\|_{L^{2}} \\
& \leq \frac{\gamma}{2}\left\|\Lambda^{\beta} \widetilde{w}\right\|_{L^{2}}^{2}+c\left\|w^{(1)}\right\|_{B_{2,1}^{1+\frac{d}{2}-\beta}}^{2}\|\widetilde{u}\|_{L^{2}}^{2},
\end{aligned}
$$

where in the last inequality we have made use of

$$
\|\widetilde{u}\|_{L^{p}} \leq c\left\|\Lambda^{\alpha} \widetilde{u}\right\|_{L^{2}}
$$

Combining the estimates leads to

$$
\frac{d}{d t}\left(\|\widetilde{u}\|_{L^{2}}^{2}+\|\widetilde{w}\|_{L^{2}}^{2}\right)+2(\nu+k)\left\|\Lambda^{\alpha} \widetilde{u}\right\|_{L^{2}}^{2}+\gamma\left\|\Lambda^{\beta} \widetilde{w}\right\|_{L^{2}}^{2}+8 k\|\widetilde{w}\|_{L^{2}}^{2}
$$




$$
\leq\left(c\left\|u^{(1)}\right\|_{B_{2,1}^{1+\frac{d}{2}}}+c\left\|w^{(1)}\right\|_{B_{2,1}^{1+\frac{d}{2}-\beta}}^{2}\right)\left(\|\widetilde{u}\|_{L^{2}}^{2}+\|\widetilde{w}\|_{L^{2}}^{2}\right)
$$

Since $u^{(1)} \in L^{1}\left(0, T, B_{2,1}^{1+\frac{d}{2}}\right)$ and $w^{(1)} \in L^{1}\left(0, T, B_{2,1}^{1+\frac{d}{2}}\right) \cap L^{\infty}\left(0, T, B_{2,1}^{1+\frac{d}{2}-2 \beta}\right)$,

$$
\begin{aligned}
\int_{0}^{T}\left\|u^{(1)}(t)\right\|_{B_{2,1}^{1+\frac{d}{2}}} d t<\infty & \\
\int_{0}^{T}\left\|w^{(1)}(t)\right\|_{B_{2,1}^{1+\frac{d}{2}-2 \beta}}^{2} d t & \leq \int_{0}^{T}\left\|w^{(1)}(t)\right\|_{B_{2,1}^{1+\frac{d}{2}}}\left\|w^{(1)}(t)\right\|_{B_{2,1}^{1+\frac{d}{2}-2 \beta}} d t \\
& \leq\left\|w^{(1)}(t)\right\|_{L^{\infty}\left(0, T, B_{2,1}^{1+\frac{d}{2}-2 \beta}\right)} \int_{0}^{T}\left\|w^{(1)}(t)\right\|_{B_{2,1}^{1+\frac{d}{2}}} d t<\infty .
\end{aligned}
$$

Applying Gronwall's inequality to (3.17) yields

$$
\|\widetilde{u}\|_{L^{2}}=\|\widetilde{w}\|_{L^{2}}=0
$$

which leads to the desired uniqueness. This completes the proof of the uniqueness part of Theorem 1.1.

\section{Proof of Theorem 1.2}

The proof of Theorem 1.2 is similar to the one of Theorem 1.1. To avoid repetitions, we will refer next to some inequalities already showed in the proof of Theorem 1.1. In this proof, we consider the system of equations (1.1) with $\beta=0$, that is

$$
\left\{\begin{array}{l}
\partial_{t} u+(\nu+k)(-\Delta)^{\alpha} u+u \cdot \nabla u+\nabla \Pi-2 k \nabla \times w=0 \\
\partial_{t} w+(4 k+\gamma) w+2 k \nabla \times u+u \cdot \nabla w=0 \\
\nabla \cdot u=0 \\
u(x, 0)=u_{0}(x), \quad w(x, 0)=w_{0}(x)
\end{array}\right.
$$

\subsection{Existence of a weak solution}

This subsection proves the existence part of Theorem 1.2. The approach is to construct a successive approximation sequence and show that the limit of a subsequence actually solves (4.1) in the weak sense.

Proof for the existence part of Theorem 1.2. We consider a successive approximation $\left\{\left(u^{(n)}, w^{(n)}\right)\right\}$ 
satisfying

$$
\left\{\begin{array}{l}
u^{(1)}=S_{2} u_{0}, \quad w^{(1)}=S_{2} w_{0} \\
\partial_{t} u^{(n+1)}+(\nu+k)\left(-\Delta^{\alpha}\right) u^{(n+1)}=\mathbb{P}\left(-u^{(n)} \cdot \nabla u^{(n+1)}\right)+2 k \nabla \times w^{(n)} \\
\partial_{t} w^{(n+1)}=-(4 k+\gamma) w^{(n+1)}-2 k \nabla \times u^{(n)}-u^{(n)} \cdot \nabla w^{(n+1)} \\
u^{(n+1)}(x, 0)=S_{n+1} u_{0}, \quad w^{(n+1)}(x, 0)=S_{n+1} w_{0}
\end{array}\right.
$$

where $\mathbb{P}=I-\nabla(-\Delta)^{-1}$ div is the standard Leray Projection. For

$$
M=2\left(\left\|u_{0}\right\|_{B_{2,1}^{1+\frac{d}{2}-2 \alpha}}+\left\|w_{0}\right\|_{B_{2,1}^{\frac{d}{2}}}\right),
$$

$T>0$ being sufficiently small and $0<\delta<1$ (to be specified later), we set

$$
\begin{gathered}
Y \equiv\left\{(u, w) \mid\|u\|_{L^{\infty}\left(0, T, B_{2,1}^{1+\frac{d}{2}-2 \alpha}\right)} \leq M,\|w\|_{L^{\infty}\left(0, T, B_{2,1}^{2}\right)} \leq M,\right. \\
\left.\|u\|_{L^{1}\left(0, T, B_{2,1}^{1+\frac{d}{2}}\right)} \leq \delta, \quad\|w\|_{L^{1}\left(0, T, B_{2,1}^{\frac{d}{2}}\right)} \leq \delta\right\} .
\end{gathered}
$$

We show that $\left\{\left(u^{(n)}, w^{(n)}\right)\right\}$ has a subsequence that converges to the weak solution of (4.1). This process consists of three main steps. The first step is to show that $\left\{\left(u^{(n)}, w^{(n)}\right)\right\}$ is uniformly bounded in $Y$. The second step is to extract a strongly convergent subsequence via the Aulin-Lions Lemma. While the last step is to show that the limit is indeed a weak solution of (4.1).

To show the uniform bound for $\left\{\left(u^{(n)}, w^{(n)}\right)\right\}$ in Y, we prove by induction. Clearly,

$$
\begin{aligned}
& \left\|u^{(1)}\right\|_{L^{\infty}\left(0, T, B_{2,1}^{1+\frac{d}{2}-2 \alpha}\right)}=\left\|S_{2} u_{0}\right\|_{L^{\infty}\left(0, T, B_{2,1}^{1+\frac{d}{2}-2 \alpha}\right)} \leq M, \\
& \left\|w^{(1)}\right\|_{L^{\infty}\left(0, T, B_{2,1}^{\frac{d}{2}}\right)}=\left\|S_{2} w_{0}\right\|_{L^{\infty}\left(0, T, B_{2,1}^{\frac{d}{2}}\right)} \leq M .
\end{aligned}
$$

If $T>0$ is sufficiently small, then

$$
\begin{gathered}
\left\|u^{(1)}\right\|_{L^{1}\left(0, T, B_{2,1}^{1+\frac{d}{2}}\right)} \leq T\left\|S_{2} u_{0}\right\|_{B_{2,1}^{1+\frac{d}{2}}} \leq T c\left\|u_{0}\right\|_{B_{2,1}^{1+\frac{d}{2}-2 \alpha}} \leq \delta, \\
\left\|w^{(1)}\right\|_{L^{1}\left(0, T, B_{2,1}^{\frac{d}{2}}\right)} \leq T\left\|S_{2} w_{0}\right\|_{B_{2,1}^{\frac{d}{2}}} \leq T c\left\|w_{0}\right\|_{B_{2,1}^{\frac{d}{2}}} \leq \delta .
\end{gathered}
$$

Assuming that $\left(u^{(n)}, w^{(n)}\right)$ obeys the bounds defined in $Y$, namely

$$
\begin{aligned}
& \left\|u^{(n)}\right\|_{L^{\infty}\left(0, T, B_{2,1}^{1+\frac{d}{2}-2 \alpha}\right)} \leq M,\left\|w^{(n)}\right\|_{L^{\infty}\left(0, T, B_{2,1}^{\frac{d}{2}}\right)} \leq M, \\
& \left\|u^{(n)}\right\|_{L^{1}\left(0, T, B_{2,1}^{1+\frac{d}{2}}\right)} \leq \delta, \quad\left\|w^{(n)}\right\|_{L^{1}\left(0, T, B_{2,1}^{\frac{d}{2}}\right)} \leq \delta .
\end{aligned}
$$

we prove that $\left\{\left(u^{(n+1)}, w^{(n+1)}\right)\right\}$ obeys the same bound for suitably selected $T>0, M>0$ and $\delta>0$. For sake of clarity, the proof of the four bounds is achieved in the following four steps. 
4.1.1 The estimate of $u^{(n+1)}$ in $L^{\infty}\left(0, T, B_{2,1}^{1+\frac{d}{2}-2 \alpha}\left(\mathbb{R}^{d}\right)\right)$. Following the same method as in the proof of the first step of Theorem 1.1, we write the inequality

$$
\begin{aligned}
\left\|u^{(n+1)}(t)\right\|_{B_{2,1}^{1+\frac{d}{2}-2 \alpha}} \leq\left\|u_{0}^{(n+1)}\right\|_{B_{2,1}^{1+\frac{d}{2}-2 \alpha}} & \\
& +\sum_{j \geq-1} 2^{\left(1+\frac{d}{2}-2 \alpha\right) j} \int_{0}^{t} e^{-c_{0} 2^{2 \alpha j}(t-\tau)}\left(J_{1}+\cdots+J_{4}\right) d \tau,
\end{aligned}
$$

where

$$
\begin{aligned}
& J_{1}=c\left\|\Delta_{j} u^{(n+1)}\right\|_{L^{2}} \sum_{m \leq j-1} 2^{\left(1+\frac{d}{2}\right) m}\left\|\Delta_{m} u^{(n)}\right\|_{L^{2}}, \\
& J_{2}=c\left\|\Delta_{j} u^{(n)}\right\|_{L^{2}} \sum_{m \leq j} 2^{\left(1+\frac{d}{2}\right) m}\left\|\Delta_{m} u^{(n+1)}\right\|_{L^{2}}, \\
& J_{3}=c 2^{j} \sum_{k \geq j-1} 2^{\frac{d}{2} k}\left\|\widetilde{\Delta}_{k} u^{(n+1)}\right\|_{L^{2}}\left\|\Delta_{k} u^{(n)}\right\|_{L^{2}}, \\
& J_{4}=c 2^{j}\left\|\Delta_{j} w^{(n)}\right\|_{L^{2}} .
\end{aligned}
$$

The terms on the right hand side can be estimated as follows. Recalling the definition of $J_{1}$ above and using the inductive assumption on $u^{(n)}$, we have for any $t \leq T$,

$$
\begin{aligned}
& \sum_{j \geq-1} 2^{\left(1+\frac{d}{2}-2 \alpha\right) j} \int_{0}^{t} e^{c_{0} 2^{2 \alpha j}(t-\tau)} J_{1} d \tau \\
& =c \int_{0}^{t} \sum_{j \geq-1} 2^{\left(1+\frac{d}{2}-2 \alpha\right) j}\left\|\Delta_{j} u^{(n+1)}\right\|_{L^{2}} \sum_{m \leq j-1} 2^{\left(1+\frac{d}{2}\right) m}\left\|\Delta_{m} u^{(n)}(\tau)\right\|_{L^{2}} d \tau
\end{aligned}
$$

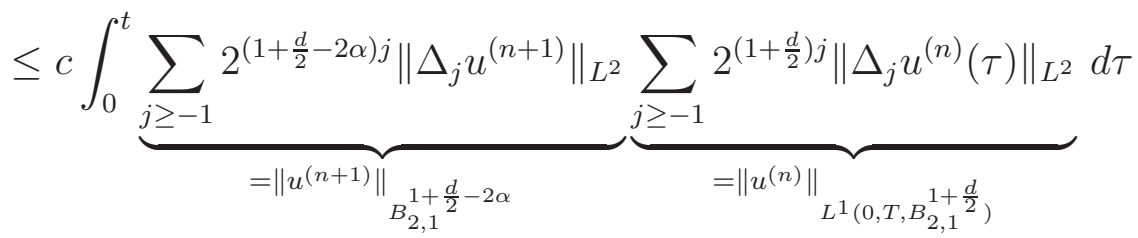

$$
\begin{aligned}
& \leq c\left\|u^{(n+1)}\right\|_{L^{\infty}\left(0, T, B_{2,1}^{1+\frac{d}{2}-2 \alpha}\right)}\left\|u^{(n)}\right\|_{L^{1}\left(0, T, B_{2,1}^{1+\frac{d}{2}}\right)} \\
& \leq c \delta\left\|u^{(n+1)}\right\|_{L^{\infty}\left(0, T, B_{2,1}^{1+\frac{d}{2}-2 \alpha}\right)} .
\end{aligned}
$$

The terms with $J_{2}$ and $J_{3}$ can be similarly estimated and obey the same bound. In fact, by Young's inequality for series convolution,

$$
\begin{aligned}
& \sum_{j \leq-1} 2^{\left(1+\frac{d}{2}-2 \alpha\right) j} \int_{0}^{t} e^{-c_{0} 2^{2 \alpha j}(t-\tau)} J_{2} d \tau \\
& \quad \leq c \int_{0}^{t} \sum_{j \geq-1} 2^{\left(1+\frac{d}{2}\right) j}\left\|\Delta_{j} u^{(n)}\right\|_{L^{2}} \sum_{m \leq j} 2^{2 \alpha(m-j)} 2^{\left(1+\frac{d}{2}-2 \alpha\right) m}\left\|\Delta_{m} u^{(n+1)}(\tau)\right\|_{L^{2}} d \tau
\end{aligned}
$$




$$
\begin{aligned}
& \leq c \int_{0}^{t}\left\|u^{(n)}(\tau)\right\|_{B_{2,1}^{1+\frac{d}{2}}}\left\|u^{(n+1)}(\tau)\right\|_{B_{2,1}^{1+\frac{d}{2}-2 \alpha}} d \tau \\
& \leq c\left\|u^{(n)}\right\|_{L^{1}\left(0, T, B_{2,1}^{1+\frac{d}{2}}\right)}\left\|u^{(n+1)}\right\|_{L^{\infty}\left(0, T, B_{2,1}^{1+\frac{d}{2}-2 \alpha}\right)} \\
& \leq c \delta\left\|u^{(n+1)}\right\|_{L^{\infty}\left(0, T, B_{2,1}^{1+\frac{d}{2}-2 \alpha}\right)} .
\end{aligned}
$$

Similarly the term with $J_{3}$ is bounded by

$$
\begin{aligned}
\sum_{j \geq-1} & 2^{\left(1+\frac{d}{2}-2 \alpha\right) j} \int_{0}^{t} e^{-c_{0} 2^{2 \alpha j}(t-\tau)} J_{3} d \tau \\
& =\int_{0}^{t} \sum_{j \geq-1} 2^{\left(1+\frac{d}{2}-2 \alpha\right) j} \sum_{k \geq j-1} 2^{j} 2^{\frac{d}{2} k}\left\|\widetilde{\Delta}_{k} u^{(n+1)}\right\|_{L^{2}}\left\|\Delta_{k} u^{(n)}\right\|_{L^{2}} d \tau \\
& =c \int_{0}^{t} \sum_{j \geq-1} \sum_{k \geq j-1} 2^{\left(2+\frac{d}{2}-2 \alpha\right)(j-k)} 2^{\left(1+\frac{d}{2}\right) k}\left\|\Delta_{k} u^{(n)}\right\|_{L^{2}} 2^{\left(1+\frac{d}{2}-2 \alpha\right) k}\left\|\widetilde{\Delta}_{k} u^{(n+1)}\right\|_{L^{2}} d \tau \\
& \leq c \int_{0}^{t}\left\|u^{(n)}(\tau)\right\|_{B_{2,1}^{1+\frac{d}{2}}}\left\|u^{(n+1)}(\tau)\right\|_{B_{2,1}^{1+\frac{d}{2}-2 \alpha}} d \tau \\
& \leq c\left\|u^{(n)}\right\|\left\|_{L^{1}\left(0, T, B_{2,1}^{1+\frac{d}{2}}\right)}\right\| u^{(n+1)} \|_{L^{\infty}\left(0, T, B_{2,1}^{1+\frac{d}{2}-2 \alpha}\right)} \\
& \leq c \delta\left\|u^{(n+1)}\right\|_{L^{\infty}\left(0, T, B_{2,1}^{1+\frac{d}{2}-2 \alpha}\right)} \cdot
\end{aligned}
$$

Now for the term with $J_{4}$ we write

$$
\begin{aligned}
\sum_{j \geq-1} 2^{\left(1+\frac{d}{2}-2 \alpha\right) j} \int_{0}^{t} e^{-c_{0} 2^{2 \alpha j(t-\tau)}} J_{4} d \tau & =\sum_{j \geq-1} 2^{\left(1+\frac{d}{2}-2 \alpha\right) j} \int_{0}^{t} e^{-c_{0} 2^{2 \alpha j(t-\tau)}} c 2^{j}\left\|\Delta_{j} w^{(n)}\right\|_{L^{2}} d \tau \\
& \leq \sum_{j \geq-1} \int_{0}^{t} c 2^{\left(2+\frac{d}{2}-2 \alpha\right) j}\left\|\Delta_{j} w^{(n)}\right\|_{L^{2}} d \tau \\
& \underbrace{\leq}_{\text {since } \alpha \geq 1} c\left\|w^{(n)}\right\|_{L^{1}\left(0, T, B_{2,1}^{\frac{d}{2}}\right)} \\
& \leq c \delta .
\end{aligned}
$$

Collecting the bounds above and inserting them in (4.4), we find for any $t \leq T$

$$
\left\|u^{(n+1)}(t)\right\|_{B_{2,1}^{1+\frac{d}{2}-2 \alpha}} \leq\left\|u_{0}^{(n+1)}\right\|_{B_{2,1}^{1+\frac{d}{2}-2 \alpha}}+c \delta\left\|u^{(n+1)}\right\|_{L^{\infty}\left(0, T, B_{2,1}^{1+\frac{d}{2}-2 \alpha}\right)}+c \delta .
$$

Therefore

$$
\left\|u^{(n+1)}(t)\right\|_{L^{\infty}\left(0, T, B_{2,1}^{1+\frac{d}{2}-2 \alpha}\right)} \leq\left\|u_{0}^{(n+1)}\right\|_{B_{2,1}^{1+\frac{d}{2}-2 \alpha}}+c \delta\left\|u^{(n+1)}\right\|_{L^{\infty}\left(0, T, B_{2,1}^{1+\frac{d}{2}-2 \alpha}\right)}+c \delta .
$$

Choosing $\delta$ such that $c \delta \leq \min \left(\frac{1}{4}, \frac{M}{4}\right)$ we get

$$
\left\|u^{(n+1)}(t)\right\|_{L^{\infty}\left(0, T, B_{2,1}^{1+\frac{d}{2}-2 \alpha}\right)} \leq \frac{M}{2}+\frac{1}{4}\left\|u^{(n+1)}\right\|_{L^{\infty}\left(0, T, B_{2,1}^{1+\frac{d}{2}-2 \alpha}\right)}+\frac{M}{4}
$$


which implies

$$
\left\|u^{(n+1)}(t)\right\|_{L^{\infty}\left(0, T, B_{2,1}^{1+\frac{d}{2}-2 \alpha}\right)} \leq M
$$

4.1.2 The estimate of $\left\|u^{(n+1)}\right\|_{L^{1}\left(0, T, B^{1+\frac{d}{2}}\right)}$. Following the same method as in the proof of the second step of Theorem 1.1, we write the inequality

$$
\begin{aligned}
\left\|u^{(n+1)}\right\|_{L^{1}\left(0, T, B_{2,1}^{1+\frac{d}{2}}\right)} \leq \int_{0}^{T} \sum_{j \geq-1} & 2^{\left(1+\frac{d}{2}\right) j} e^{-c_{0} 2^{2 \alpha j} t}\left\|\Delta_{j} u_{0}^{(n+1)}\right\|_{L^{2}} d t \\
& \quad+\int_{0}^{T} \int_{0}^{s} \sum_{j \geq-1} 2^{\left(1+\frac{d}{2}\right) j} e^{-c_{0} 2^{2 \alpha j}(s-\tau)}\left(J_{1}+\cdots+J_{4}\right) d \tau d s .
\end{aligned}
$$

We estimate the terms on the right and start with the first term.

$$
\int_{0}^{T} \sum_{j \geq-1} 2^{\left(1+\frac{d}{2}\right) j} e^{-c_{0} 2^{2 \alpha j} t}\left\|\Delta_{j} u_{0}^{(n+1)}\right\|_{L^{2}} d t=c \sum_{j \geq-1} 2^{\left(1+\frac{d}{2}-2 \alpha\right) j}\left(1-e^{-c_{0} 2^{2 \alpha j} T}\right)\left\|\Delta_{j} u_{0}^{(n+1)}\right\|_{L^{2}} .
$$

Since $u_{0} \in B_{2,1}^{1+\frac{d}{2}-2 \alpha}$, then by the Dominated Convergence Theorem

$$
\lim _{T \rightarrow 0} \sum_{j \geq-1} 2^{\left(1+\frac{d}{2}-2 \alpha\right) j}\left(1-e^{-c_{0} 2^{2 \alpha j} T}\right)\left\|\Delta_{j} u_{0}^{(n+1)}\right\|_{L^{2}}=0
$$

Therefore, we can choose $T$ sufficiently small such that

$$
\int_{0}^{T} \sum_{j \geq-1} 2^{\left(1+\frac{d}{2}\right) j} e^{-c_{0} 2^{2 \alpha j} t}\left\|\Delta_{j} u_{0}^{(n+1)}\right\|_{L^{2}} \leq \frac{\delta}{4}
$$

Applying Young's inequality for the time convolution, we have

$$
\begin{aligned}
& \int_{0}^{T} \sum_{j \geq-1} 2^{\left(1+\frac{d}{2}\right) j} \int_{0}^{s} e^{-c_{0} 2^{2 \alpha j}(s-\tau)} J_{1} d \tau d s \\
& \quad=c \int_{0}^{T} \sum_{j \geq-1} 2^{\left(1+\frac{d}{2}\right) j} \int_{0}^{s} e^{-c_{0} 2^{2 \alpha j}(s-\tau)}\left\|\Delta_{j} u^{(n+1)}(\tau)\right\|_{L^{2}} \sum_{m \leq j-1} 2^{\left(1+\frac{d}{2}\right) m}\left\|\Delta_{m} u^{(n)}\right\|_{L^{2}} d \tau d s \\
& \quad \leq c \sum_{j \geq-1} 2^{\left(1+\frac{d}{2}\right) j} \int_{0}^{T}\left\|\Delta_{j} u^{(n+1)}(\tau)\right\|_{L^{2}} \sum_{m \leq j-1} 2^{\left(1+\frac{d}{2}\right) m}\left\|\Delta_{m} u^{(n)}\right\|_{L^{2}} d \tau \int_{0}^{T} e^{-c_{0} 2^{2 \alpha j} s} d s .
\end{aligned}
$$

Then, using the fact that there exists $c_{2}>0$ satisfying for $j \geq 0$,

$$
\int_{0}^{T} e^{-c_{0} 2^{2 \alpha j} s} d s \leq c 2^{-2 \alpha j}\left(1-e^{-c_{2} T}\right)
$$


we get

$$
\begin{aligned}
\int_{0}^{T} \sum_{j \geq-1} 2^{\left(1+\frac{d}{2}\right) j} & \int_{0}^{s} e^{-c_{0} 2^{2 \alpha j}(s-\tau)} J_{1} d \tau d s \\
& \leq c\left(1-e^{-c_{2} T}\right) \int_{0}^{T} \sum_{j \geq-1} 2^{\left(1+\frac{d}{2}-2 \alpha\right) j}\left\|\Delta_{j} u^{(n+1)}(\tau)\right\|_{L^{2}} \sum_{m \leq j-1} 2^{\left(1+\frac{d}{2}\right) m}\left\|\Delta_{m} u^{(m)}\right\|_{L^{2}} d \tau \\
& \leq c\left(1-e^{-c_{2} T}\right) \int_{0}^{T} \sum_{j \geq-1} 2^{\left(1+\frac{d}{2}-2 \alpha\right) j}\left\|\Delta_{j} u^{(n+1)}(\tau)\right\|_{L^{2}} \sum_{j \geq-1} 2^{\left(1+\frac{d}{2}\right) j}\left\|\Delta_{j} u^{(n)}\right\|_{L^{2}} d \tau \\
& \leq c\left(1-e^{-c_{2} T}\right)\left\|u^{(n+1)}\right\|_{L^{\infty}\left(0, T, B_{2,1}^{1+\frac{d}{2}-2 \alpha}\right)} \underbrace{\left\|u^{(n)}\right\|_{L^{1}\left(0, T, B_{2,1}+\frac{d}{2}\right)}}_{\leq \delta} \\
& \leq c \delta\left(1-e^{-c_{2} T}\right)\left\|u^{(n+1)}\right\| \|_{L^{\infty}\left(0, T, B_{2,1}^{1+\frac{d}{2}-2 \alpha}\right)},{ }
\end{aligned}
$$

The terms with $J_{2}$ and $J_{3}$ can be similarly estimated and obey the same bound.

$$
\begin{aligned}
& \int_{0}^{T} \sum_{j \geq-1} 2^{\left(1+\frac{d}{2}\right) j} \int_{0}^{s} e^{-c_{0} 2^{2 \alpha j}(s-\tau)} J_{2} d \tau d s \\
& \quad=c \int_{0}^{T} \sum_{j \geq-1} 2^{\left(1+\frac{d}{2}\right) j} \int_{0}^{s} e^{-c_{0} 2^{2 \alpha j}(s-\tau)}\left\|\Delta_{j} u^{(n)}\right\|_{L^{2}} \sum_{m \leq j} 2^{\left(1+\frac{d}{2}\right) m}\left\|\Delta_{m} u^{(n+1)}\right\|_{L^{2}} d \tau d s \\
& \quad \leq c \sum_{j \geq-1} 2^{\left(1+\frac{d}{2}\right) j} \int_{0}^{T}\left\|\Delta_{j} u^{(n)}\right\|_{L^{2}} \sum_{m \leq j} 2^{\left(1+\frac{d}{2}\right) m}\left\|_{\Delta_{m}} u^{(n+1)}\right\|_{L^{2}} d \tau \int_{0}^{T} e^{-c_{0} 2^{2 \alpha j} s} d s .
\end{aligned}
$$

Hence due to (4.6)

$$
\begin{aligned}
\int_{0}^{T} & \sum_{j \geq-1} 2^{\left(1+\frac{d}{2}\right) j} \int_{0}^{s} e^{-c_{0} 2^{2 \alpha j}(s-\tau)} J_{2} d \tau d s \\
& \leq c\left(1-e^{-c_{2} T}\right) \int_{0}^{T} \sum_{j \geq-1} 2^{\left(1+\frac{d}{2}-2 \alpha\right) j}\left\|\Delta_{j} u^{(n)}\right\|_{L^{2}} \sum_{m \leq j-1} 2^{\left(1+\frac{d}{2}\right) m}\left\|\Delta_{m} u^{(n+1)}\right\|_{L^{2}} d \tau \\
& \leq c\left(1-e^{-c_{2} T}\right) \int_{0}^{T} \sum_{j \geq-1} 2^{\left(1+\frac{d}{2}-2 \alpha\right) j}\left\|\Delta_{j} u^{(n+1)}\right\|_{L^{2}} \sum_{j \geq-1} 2^{\left(1+\frac{d}{2}\right) j}\left\|\Delta_{j} u^{(n)}\right\|_{L^{2}} d \tau \\
& \leq c\left(1-e^{-c_{2} T}\right)\left\|u^{(n+1)}\right\|_{L^{\infty}\left(0, T, B_{2,1}^{1+\frac{d}{2}-2 \alpha}\right)}\left\|u^{(n)}\right\|_{L^{1}\left(0, T, B_{2,1}^{1+\frac{d}{2}}\right)} \\
& \leq c\left(1-e^{-c_{2} T}\right) \delta\left\|u^{(n+1)}\right\|_{L^{\infty}\left(0, T, B_{2,1}^{1+\frac{d}{2}-2 \alpha}\right)} \cdot
\end{aligned}
$$

Similarly

$$
\int_{0}^{T} \sum_{j \geq-1} 2^{\left(1+\frac{d}{2}\right) j} \int_{0}^{s} e^{-c_{0} 2^{2 \alpha j}(s-\tau)} J_{3} d \tau d s
$$




$$
\begin{aligned}
& =c \int_{0}^{T} \sum_{j \geq-1} 2^{\left(2+\frac{d}{2}\right) j} \int_{0}^{s} e^{-c_{0} 2^{2 \alpha j}(s-\tau)} \sum_{k \geq j-1} 2^{\frac{d}{2} k}\left\|\Delta_{k} u^{(n)}\right\|_{L^{2}}\left\|\widetilde{\Delta}_{k} u^{(n+1)}\right\|_{L^{2}} d \tau d s \\
& \leq c \sum_{j \geq-1} 2^{\left(2+\frac{d}{2}\right) j} \int_{0}^{T} \sum_{k \geq j-1} 2^{\frac{d}{2} k}\left\|\Delta_{k} u^{(n)}\right\|_{L^{2}}\left\|\widetilde{\Delta}_{k} u^{(n+1)}\right\|_{L^{2}} d \tau \int_{0}^{T} e^{-c_{0} 2^{2 \alpha j} s} d s .
\end{aligned}
$$

By (4.6) and the inequality above,

$$
\begin{aligned}
& \int_{0}^{T} \sum_{j \geq-1} 2^{\left(1+\frac{d}{2}\right) j} \int_{0}^{s} e^{-c_{0} 2^{2 \alpha j}(s-\tau)} J_{3} d \tau d s \\
& \leq c\left(1-e^{-c_{2} T}\right) \int_{0}^{T} \sum_{j \geq-1} 2^{\left(2+\frac{d}{2}-2 \alpha\right) j} \sum_{k \geq j-1} 2^{\frac{d}{2} k}\left\|\Delta_{k} u^{(n)}\right\|_{L^{2}}\left\|\widetilde{\Delta}_{k} u^{(n+1)}\right\|_{L^{2}} d \tau \\
& \leq c\left(1-e^{-c_{2} T}\right) \int_{0}^{T} \sum_{j \geq-1} 2^{\left(2+\frac{d}{2}-2 \alpha\right) j}\left\|\widetilde{\Delta}_{j} u^{(n+1)}\right\|_{L^{2}} \sum_{j \geq-1} 2^{\left(\frac{d}{2}\right) j}\left\|\Delta_{j} u^{(n)}\right\|_{L^{2}} d \tau \\
& \leq c\left(1-e^{-c_{2} T}\right) \int_{0}^{T} \sum_{j \geq-1} 2^{\left(1+\frac{d}{2}-2 \alpha\right) j}\left\|\widetilde{\Delta}_{j} u^{(n+1)}\right\|_{L^{2}} \sum_{j \geq-1} 2^{\left(1+\frac{d}{2}\right) j}\left\|\Delta_{j} u^{(n)}\right\|_{L^{2}} d \tau \\
& \leq c\left(1-e^{-c_{2} T}\right)\left\|u^{(n+1)}\right\|_{L^{\infty}\left(0, T, B_{2,1}^{1+\frac{d}{2}-2 \alpha}\right)}\left\|u^{(n)}\right\|_{L^{1}\left(0, T, B_{2,1}^{1+\frac{d}{2}}\right)} \\
& \leq c\left(1-e^{-c_{2} T}\right) \delta\left\|u^{(n+1)}\right\|_{L^{\infty}\left(0, T, B_{2,1}^{1+\frac{d}{2}-2 \alpha}\right)} .
\end{aligned}
$$

The term with $J_{4}$ is bounded by

$$
\begin{aligned}
\int_{0}^{T} \sum_{j \geq-1} 2^{\left(1+\frac{d}{2}\right) j} & \int_{0}^{s} e^{-c_{0} 2^{2 \alpha j}(s-\tau)} J_{4} d \tau d s \\
& =c \int_{0}^{T} \sum_{j \geq-1} 2^{\left(2+\frac{d}{2}\right) j} \int_{0}^{s} e^{-c_{0} 2^{2 \alpha j}(s-\tau)}\left\|\Delta_{j} w^{(n)}\right\|_{L^{2}} d \tau d s \\
& \leq c \sum_{j \geq-1} 2^{\left(2+\frac{d}{2}\right) j} \int_{0}^{T}\left\|\Delta_{j} w^{(n)}\right\|_{L^{2}} d \tau \int_{0}^{T} e^{-c_{0} 2^{2 \alpha j} s} d s \\
& \leq c\left(1-e^{-c_{2} T}\right) \int_{0}^{T} \sum_{j \geq-1} 2^{\left(2+\frac{d}{2}-2 \alpha\right)}\left\|\Delta_{j} w^{(n)}\right\|_{L^{2}} d \tau \\
& \left.\leq e^{-c_{2} T}\right) \int_{0}^{T} \sum_{j \geq-1} 2^{\left(\frac{d}{2}\right) j}\left\|\Delta_{j} w^{(n)}\right\|_{L^{2}} d \tau \\
& =c\left(1-e^{-c_{2} T}\right)\left\|w^{(n)}\right\|_{L^{1}\left(0, T, B_{2,1}\right)}^{\left.\frac{d}{2}\right)}
\end{aligned}
$$

Collecting the estimates above and inserting them in (4.5) leads to

$$
\left\|u^{(n+1)}\right\|_{L^{1}\left(0, T, B_{2,1}^{1+\frac{d}{2}}\right)} \leq \frac{\delta}{4}+c \delta\left(1-e^{-c_{2} T}\right)\left\|u^{(n+1)}\right\|_{L^{\infty}\left(0, T, B_{2,1}^{1+\frac{d}{2}-2 \alpha}\right)}
$$




$$
\begin{gathered}
+c\left(1-e^{-c_{2} T}\right)\left\|w^{(n)}\right\|_{L^{1}\left(0, T, B_{2,1}^{\frac{d}{2}}\right)} \\
\leq \frac{\delta}{4}+c \delta\left(1-e^{-c_{2} T}\right) M+c\left(1-e^{-c_{2} T}\right) \delta .
\end{gathered}
$$

Choosing $\mathrm{T}$ sufficiently small such that $c\left(1-e^{-c_{2} T}\right) \leq \min \left(\frac{1}{4 M}, \frac{1}{2}\right)$ we get

$$
\left\|u^{(n+1)}\right\|_{L^{1}\left(0, T, B_{2,1}^{1+\frac{d}{2}}\right)} \leq \frac{\delta}{4}+\frac{\delta}{4}+\frac{\delta}{2}=\delta
$$

4.1.3 The estimate of $w^{(n+1)}$ in $L^{\infty}\left(0, T, B_{2,1}^{\frac{d}{2}}\left(\mathbb{R}^{d}\right)\right)$. Applying $\Delta_{j}$ to the third equation in (4.2) and then dotting with $\Delta_{j} w^{(n+1)}$, we obtain

$$
\begin{aligned}
\frac{1}{2} \frac{d}{d t}\left\|\Delta_{j} w^{(n+1)}\right\|_{L^{2}}^{2}+(4 k+\gamma)\left\|\Delta_{j} w^{(n+1}\right\|_{L^{2}}^{2} & =-2 k \int \Delta_{j}\left(\nabla \times u^{(n)}\right) \Delta_{j} w^{(n+1)} d x \\
& -\int \Delta_{j}\left(u^{(n)} \cdot \nabla w^{(n+1)}\right) \Delta_{j} w^{(n+1)} d x \\
& =B_{1}+B_{2},
\end{aligned}
$$

where

$$
\begin{aligned}
& B_{1}=-2 k \int \Delta_{j}\left(\nabla \times u^{(n)}\right) \Delta_{j} w^{(n+1)} d x, \\
& B_{2}=-\int \Delta_{j}\left(u^{(n)} \cdot \nabla w^{(n+1)}\right) \Delta_{j} w^{(n+1)} d x .
\end{aligned}
$$

By Hölder's inequality and Bernstein's inequality

$$
\begin{aligned}
\left|B_{1}\right| & =\left|-2 k \int \Delta_{j}\left(\nabla \times u^{(n)}\right) \Delta_{j} w^{(n+1)} d x\right| \\
& \leq 2 k\left\|\Delta_{j}\left(\nabla \times u^{(n)}\right)\right\|_{L^{2}}\left\|\Delta_{j} w^{(n+1)} d x\right\|_{L^{2}} \\
& \leq c 2^{j}\left\|\Delta_{j} u^{(n)}\right\|_{L^{2}}\left\|\Delta_{j} w^{(n+1)}\right\|_{L^{2}} .
\end{aligned}
$$

By Lemma 2.6,

$$
\begin{aligned}
\left|B_{2}\right| & =\left|-\int \Delta_{j}\left(u^{(n)} \cdot \nabla w^{(n+1)}\right) \Delta_{j} w^{(n+1)} d x\right| \\
& \leq c\left\|\Delta_{j} w^{(n+1)}\right\|_{L^{2}}^{2} \sum_{m \leq j-1} 2^{\left(1+\frac{d}{2}\right) m}\left\|\Delta_{m} u^{(n)}\right\|_{L^{2}} \\
& +c\left\|\Delta_{j} w^{(n+1)}\right\|_{L^{2}}\left\|\Delta_{j} u^{(n)}\right\|_{L^{2}} \sum_{m \leq j} 2^{\left(1+\frac{d}{2}\right) m}\left\|\Delta_{m} w^{(n+1)}\right\|_{L^{2}} \\
& +c\left\|\Delta_{j} w^{(n+1)}\right\|_{L^{2}} 2^{j} \sum_{k \leq j-1} 2^{\frac{d}{2} k}\left\|\widetilde{\Delta}_{k} w^{(n+1)}\right\|_{L^{2}}\left\|\Delta_{k} u^{(n)}\right\|_{L^{2}} .
\end{aligned}
$$


Inserting the estimates above in (4.7) and eliminating $\left\|\Delta_{j} w^{(n+1)}\right\|_{L^{2}}$ from both sides of the inequality, we obtain

$$
\begin{aligned}
\frac{d}{d t}\left\|\Delta_{j} w^{(n+1)}\right\|_{L^{2}}+(8 k+2 \gamma)\left\|\Delta_{j} w^{(n+1)}\right\|_{L^{2}} \leq & c 2^{j}\left\|\Delta_{j} u^{(n)}\right\|_{L^{2}} \\
& +c\left\|\Delta_{j} w^{(n+1)}\right\|_{L^{2}} \sum_{m \leq j-1} 2^{\left(1+\frac{d}{2}\right) m}\left\|\Delta_{m} u^{(n)}\right\|_{L^{2}} \\
& +c\left\|\Delta_{j} u^{(n)}\right\|_{L^{2}} \sum_{m \leq j} 2^{\left(1+\frac{d}{2}\right) m}\left\|\Delta_{m} w^{(n+1)}\right\|_{L^{2}} \\
& +c 2^{j} \sum_{k \geq j-1} 2^{\frac{d}{2} k}\left\|\widetilde{\Delta}_{k} w^{(n+1)}\right\|_{L^{2}}\left\|\Delta_{k} u^{(n)}\right\|_{L^{2}} .
\end{aligned}
$$

Integrating (4.8) in time yields, for any $t \leq T$,

$$
\left\|\Delta_{j} w^{(n+1)}\right\|_{L^{2}} \leq e^{-(8 k+2 \gamma) t}\left\|\Delta_{j} w_{0}^{(n+1)}\right\|_{L^{2}}+\int_{0}^{t} e^{-(8 k+2 \gamma)(t-\tau)}\left(K_{1}+\cdots+K_{4}\right) d \tau,
$$

where

$$
\begin{aligned}
& K_{1}=c 2^{j}\left\|\Delta_{j} u^{(n)}\right\|, \\
& K_{2}=c\left\|\Delta_{j} w^{(n+1)}\right\|_{L^{2}} \sum_{m \leq j-1} 2^{\left(1+\frac{d}{2}\right) m}\left\|\Delta_{m} u^{(n)}\right\|_{L^{2}}, \\
& K_{3}=c\left\|\Delta_{j} u^{(n)}\right\|_{L^{2}} \sum_{m \leq j} 2^{\left(1+\frac{d}{2}\right) m}\left\|\Delta_{m} w^{(n+1)}\right\|_{L^{2}}, \\
& K_{4}=c 2^{j} \sum_{k \geq j-1} 2^{\frac{d}{2} k}\left\|\widetilde{\Delta}_{k} w^{(n+1)}\right\|_{L^{2}}\left\|\Delta_{k} u^{(n)}\right\|_{L^{2}} .
\end{aligned}
$$

We multiply (4.9) by $2^{\left(\frac{d}{2}\right) j}$ and sum over $j$ to get

$$
\left\|w^{(n+1)}\right\|_{B_{2,1}^{\frac{d}{2}}} \leq\left\|w_{0}^{(n+1)}\right\|_{B_{2,1}^{\frac{d}{2}}}+\sum_{j \geq-1} \int_{0}^{t} e^{-(8 k+2 \gamma)(t-\tau)} 2^{2^{\left(\frac{d}{2}\right) j}}\left(K_{1}+\cdots+K_{4}\right) d \tau .
$$

The term with $K_{1}$ is bounded by

$$
\begin{aligned}
\sum_{j \geq-1} 2^{\frac{d}{2} j} \int_{0}^{t} e^{-(8 k+2 \gamma)(t-\tau)} K_{1} d \tau & \leq \int_{0}^{t} \sum_{j \geq-1} c 2^{\left(1+\frac{d}{2}\right) j}\left\|\Delta_{j} u^{(n)}(\tau)\right\|_{L^{2}} d \tau \\
& \leq c\left\|u^{(n)}\right\|_{L^{1}\left(0, T, B_{2,1}^{1+\frac{d}{2}}\right)} .
\end{aligned}
$$

The terms with $K_{2}$ through $K_{4}$ can be bounded suitably and obey the same bound. In fact, for the term involving $K_{2}$ we write

$$
\sum_{j \geq-1} 2^{\left(\frac{d}{2}\right) j} \int_{0}^{t} e^{-(8 k+2 \gamma)(t-\tau)} c\left\|\Delta_{j} w^{(n+1)}(\tau)\right\|_{L^{2}} \sum_{m \leq j-1} 2^{\left(1+\frac{d}{2}\right) m}\left\|\Delta_{m} u^{(n)}(\tau)\right\|_{L^{2}} d \tau
$$




$$
\begin{aligned}
& \leq c \int_{0}^{t} \sum_{j \geq-1} 2^{\left(\frac{d}{2}\right) j}\left\|\Delta_{j} w^{(n+1)}(\tau)\right\|_{L^{2}} \sum_{m \leq j-1} 2^{\left(1+\frac{d}{2}\right) m}\left\|\Delta_{m} u^{(n)}(\tau)\right\|_{L^{2}} d \tau \\
& \leq c \int_{0}^{t}\left\|w^{(n+1)}\right\|_{B_{2,1}^{\frac{d}{2}}}\left\|u^{(n)}\right\|_{B_{2,1}^{1+\frac{d}{2}}} d \tau \\
& \leq c\left\|w^{(n+1)}\right\|_{L^{\infty}\left(0, T, B_{2,1}^{\frac{d}{2}}\right)}\left\|u^{(n)}\right\|_{L^{1}\left(0, T, B_{2,1}^{1+\frac{d}{2}}\right)} .
\end{aligned}
$$

Similarly the term with $K_{3}$ is bounded by

$$
\begin{aligned}
& \sum_{j \geq-1} 2^{\left(\frac{d}{2}\right) j} \int_{0}^{t} e^{-(8 k+2 \gamma)(t-\tau)} c\left\|\Delta_{j} u^{(n)}\right\|_{L^{2}} \sum_{m \leq j} 2^{\left(1+\frac{d}{2}\right) m}\left\|\Delta_{m} w^{(n+1)}\right\|_{L^{2}} d \tau \\
& \quad \leq c \int_{0}^{t} \sum_{j \geq-1} 2^{\left(\frac{d}{2}\right) j}\left\|\Delta_{j} u^{(n)}\right\|_{L^{2}} \sum_{j \geq-1} 2^{\left(1+\frac{d}{2}\right) j}\left\|\Delta_{j} w^{(n+1)}\right\|_{L^{2}} d \tau \\
& \leq c\left\|w^{(n+1)}\right\|_{L^{\infty}\left(0, T, B_{2,1}^{\frac{d}{2}}\right)}\left\|u^{(n)}\right\|_{L^{1}\left(0, T, B_{2,1}^{1+\frac{d}{2}}\right)} .
\end{aligned}
$$

For the term with $K_{4}$ we write

$$
\begin{aligned}
& \sum_{j \geq-1} 2^{\left(\frac{d}{2}\right) j} \int_{0}^{t} c 2^{j} \sum_{k \geq j-1}\left\|\widetilde{\Delta}_{k} w^{(n+1)}\right\|_{L^{2}}\left\|\Delta_{k} u^{(n)}\right\|_{L^{2}} 2^{\frac{d k}{2}} d \tau \\
& \quad \leq c \int_{0}^{t} \sum_{j \geq-1} 2^{\left(1+\frac{d}{2}\right) j}\left\|\Delta_{j} u^{(n)}\right\|_{L^{2}} \sum_{j \geq-1} 2^{\left(\frac{d}{2}\right) j}\left\|\widetilde{\Delta}_{j} w^{(n+1)}\right\|_{L^{2}} d \tau \\
& \leq c\left\|w^{(n+1)}\right\|_{L^{\infty}\left(0, T, B_{2,1}^{\frac{d}{2}}\right)}\left\|u^{(n)}\right\|_{L^{1}\left(0, T, B_{2,1}^{1+\frac{d}{2}}\right)} .
\end{aligned}
$$

Collecting the estimates and inserting them in (4.10), we obtain for any $t \leq T$

$$
\begin{aligned}
\left\|w^{(n+1)}(t)\right\|_{B_{2,1}^{\frac{d}{2}}} & \leq\left\|w_{0}^{(n+1)}\right\|_{B_{2,1}^{\frac{d}{2}}}+c\left\|u^{(n)}\right\|_{L^{1}\left(0, T, B_{2,1}^{1+\frac{d}{2}}\right)}+c\left\|w^{(n+1)}\right\|_{L^{\infty}\left(0, T, B_{2,1}^{\frac{d}{2}}\right)}\left\|u^{(n)}\right\|_{L^{1}\left(0, T, B_{2,1}^{1+\frac{d}{2}}\right)} \\
& \leq\left\|w_{0}^{(n+1)}\right\|_{B_{2,1}^{\frac{d}{2}}}+c \delta+c \delta\left\|w^{(n+1)}\right\|_{L^{\infty}\left(0, T, B_{2,1}^{\frac{d}{2}}\right)} \cdot
\end{aligned}
$$

Therefore

$$
\left\|w^{(n+1)}(t)\right\|_{L^{\infty}\left(0, T, B_{2,1}^{\frac{d}{2}}\right)} \leq\left\|w_{0}^{(n+1)}\right\|_{B_{2,1}^{\frac{d}{2}}}+c \delta+c \delta\left\|w^{(n+1)}\right\|_{L^{\infty}\left(0, T, B_{2,1}^{\frac{d}{2}}\right)} .
$$

Choosing $c \delta \leq \min \left(\frac{1}{4}, \frac{M}{4}\right)$ we get

$$
\left\|w^{(n+1)}(t)\right\|_{L^{\infty}\left(0, T, B_{2,1}^{\frac{d}{2}}\right)} \leq \frac{M}{2}+\frac{M}{4}+\frac{1}{4}\left\|w^{(n+1)}(t)\right\|_{L^{\infty}\left(0, T, B_{2,1}^{\frac{d}{2}}\right)},
$$

which implies

$$
\left\|w^{(n+1)}(t)\right\|_{L^{\infty}\left(0, T, B_{2,1}^{\frac{d}{2}}\right)} \leq M .
$$


4.1.4 The estimate of $\left\|w^{(n+1)}(t)\right\|_{L^{1}\left(0, T, B_{2,1}^{\frac{d}{2}}\right)}$. We multiply (4.9) by $2^{\left(\frac{d}{2}\right) j}$, sum over $j$ and integrate in time to get

$$
\begin{aligned}
\left\|w^{(n+1)}\right\|_{L^{1}\left(0, T, B_{2,1}^{2}\right)} \leq & \int_{0}^{T} \sum_{j \geq-1} 2^{\frac{d}{2} j} e^{-(8 k+2 \gamma) t}\left\|\Delta_{j} w_{0}^{(n+1)}\right\|_{L^{2}} d t \\
& +\int_{0}^{T} \sum_{j \geq-1} 2^{\frac{d}{2} j} \int_{0}^{s} e^{-(8 k+2 \gamma)(s-\tau)}\left(K_{1}+\cdots+K_{4}\right) d \tau d s .
\end{aligned}
$$

Clearly

$$
\int_{0}^{T} \sum_{j \geq-1} 2^{\frac{d}{2} j} e^{-(8 k+2 \gamma) t}\left\|\Delta_{j} w_{0}^{(n+1)}\right\|_{L^{2}} d t=c \sum_{j \geq-1} 2^{\frac{d}{2} j}\left(1-e^{-(8 k+2 \gamma) T}\right)\left\|\Delta_{j} w_{0}^{(n+1)}\right\|_{L^{2}} .
$$

Since $w_{0} \in B_{2,1}^{\frac{d}{2}}$, it follows from the Dominated Convergence Theorem that

$$
\lim _{T \rightarrow 0} \sum_{j \geq-1} 2^{\frac{d}{2} j}\left(1-e^{-(8 k+2 \gamma) T}\right)\left\|\Delta_{j} w_{0}^{(n+1)}\right\|_{L^{2}}=0 .
$$

Therefore, we can choose $T$ sufficiently small such that

$$
\int_{0}^{T} \sum_{j \geq-1} 2^{\frac{d}{2} j} e^{-(8 k+2 \gamma) t}\left\|\Delta_{j} w_{0}^{(n+1)}\right\|_{L^{2}} d t \leq \frac{\delta}{2}
$$

Applying the Young's inequality for the time convolution, the term with $K_{1}$ is bounded by

$$
\begin{aligned}
\int_{0}^{T} \sum_{j \geq-1} 2^{\frac{d}{2} j} & \int_{0}^{s} e^{-(8 k+2 \gamma)(s-\tau)} K_{1} d \tau d s \\
& =c \int_{0}^{T} \sum_{j \geq-1} 2^{\left(1+\frac{d}{2}\right) j} \int_{0}^{s} e^{-(8 k+2 \gamma)(s-\tau)}\left\|\Delta_{j} u^{(n)}\right\|_{L^{2}} d \tau d s \\
& \leq\left(c \sum_{j \geq-1} 2^{\left(1+\frac{d}{2}\right) j} \int_{0}^{T}\left\|\Delta_{j} u^{(n)}\right\|_{L^{2}} d \tau\right)\left(\int_{0}^{T} e^{-(8 k+2 \gamma) s} d s\right) \\
& \leq c\left(1-e^{-(8 k+2 \gamma) T}\right) \underbrace{\int_{0}^{T} \sum_{j \geq-1} 2^{\left(1+\frac{d}{2}\right) j}\left\|\Delta_{j} u^{(n)}\right\|_{L^{2}} d \tau}_{=\left\|u^{(n)}\right\|_{L^{1}\left(0, T, B_{2,1}^{1+\frac{d}{2}}\right.} \leq \delta} \\
& \leq c\left(1-e^{-(8 k+2 \gamma) T}\right) \delta .
\end{aligned}
$$

Similarly by applying Young's inequality for the time convolution the term with $K_{2}$ is bounded by

$$
\int_{0}^{T} \sum_{j \geq-1} 2^{\frac{d}{2} j} \int_{0}^{s} e^{-(8 k+2 \gamma)(s-\tau)} K_{2} d \tau d s
$$




$$
\begin{aligned}
& =c \int_{0}^{T} \sum_{j \geq-1} 2^{\frac{d}{2} j} \int_{0}^{s} e^{-(8 k+2 \gamma)(s-\tau)}\left\|\Delta_{j} w^{(n+1)}\right\|_{L^{2}} \sum_{m \leq j-1} 2^{\left(1+\frac{d}{2}\right) m}\left\|\Delta_{m} u^{(n)}\right\|_{L^{2}} d \tau d s \\
& \leq\left(c \sum_{j \leq-1} 2^{\frac{d}{2} j} \int_{0}^{T}\left\|\Delta_{j} w^{(n+1)}\right\|_{L^{2}} \sum_{m \leq j-1} 2^{\left(1+\frac{d}{2}\right) m}\left\|\Delta_{m} u^{(n)}\right\|_{L^{2}} d \tau\right)\left(\int_{0}^{T} e^{-(8 k+2 \gamma) s} d s\right) \\
& \leq c\left(1-e^{-(8 k+2 \gamma) T}\right) \int_{0}^{T} \sum_{j \leq-1} 2^{\frac{d}{2} j}\left\|\Delta_{j} w^{(n+1)}\right\|_{L^{2}} \sum_{m \leq j-1} 2^{\left(1+\frac{d}{2}\right) m}\left\|\Delta_{m} u^{(n)}\right\|_{L^{2}} d \tau \\
& \leq c\left(1-e^{-(8 k+2 \gamma) T}\right) \int_{0}^{T} \sum_{j \geq-1} 2^{\frac{d}{2} j}\left\|\Delta_{j} w^{(n+1)}\right\|_{L^{2}} \sum_{j \geq-1} 2^{\left(1+\frac{d}{2}\right) j}\left\|\Delta_{j} u^{(n)}\right\|_{L^{2}} d \tau \\
& \leq c\left(1-e^{-(8 k+2 \gamma) T}\right)\left\|w^{(n+1)}\right\|_{L^{\infty}\left(0, T, B_{2,1}^{\frac{d}{2}}\right)}\left\|u^{(n)}\right\|_{L^{1}\left(0, T, B_{2,1}^{1+\frac{d}{2}}\right)} \\
& \leq c\left(1-e^{-(8 k+2 \gamma) T}\right) \delta M \text {. }
\end{aligned}
$$

Applying Young's inequality for the time convolution, the term with $K_{3}$ is bounded by

$$
\begin{aligned}
& \int_{0}^{T} \sum_{j \geq-1} 2^{\frac{d}{2} j} \int_{0}^{s} e^{-(8 k+2 \gamma)(s-\tau)} K_{3} d \tau d s \\
& =c\left(\int_{0}^{T} \sum_{j \geq-1} 2^{\frac{d}{2} j} \int_{0}^{s} e^{-(8 k+2 \gamma)(s-\tau)}\left\|\Delta_{j} u^{(n)}\right\|_{L^{2}}\right)\left(\sum_{m \leq j} 2^{\left(1+\frac{d}{2}\right) m}\left\|\Delta_{m} w^{(n+1)}\right\|_{L^{2}}\right) d \tau d s \\
& \leq\left(c \sum_{j \geq-1} 2^{\frac{d}{2} j} \int_{0}^{T}\left\|\Delta_{j} u^{(n)}\right\|_{L^{2}} \sum_{m \leq j} 2^{\left(1+\frac{d}{2}\right) m}\left\|\Delta_{m} w^{(n+1)}\right\|_{L^{2}} d \tau\right)\left(\int_{0}^{T} e^{-(8 k+2 \gamma) s} d s\right) \\
& \leq c\left(1-e^{-(8 k+2 \gamma) T}\right) \int_{0}^{T} \sum_{j \geq-1} 2^{\frac{d}{2} j}\left\|\Delta_{j} u^{(n)}\right\|_{L^{2}} \sum_{m \leq j} 2^{\left(1+\frac{d}{2}\right) m}\left\|\Delta_{m} w^{(n+1)}\right\|_{L^{2}} d \tau \\
& \leq c\left(1-e^{-(8 k+2 \gamma) T}\right) \int_{0}^{T} \sum_{j \geq-1} 2^{\left(1+\frac{d}{2}\right) j}\left\|\Delta_{j} u^{(n)}\right\|_{L^{2}} \sum_{j \geq-1} 2^{\frac{d}{2} j}\left\|\Delta_{j} w^{(n+1)}\right\|_{L^{2}} d \tau \\
& \leq c\left(1-e^{-(8 k+2 \gamma) T}\right)\left\|w^{(n+1)}\right\|_{L^{\infty}\left(0, T, B_{2,1}^{\left.\frac{d}{2}\right)}\right.}\left\|u^{(n)}\right\|_{L^{1}\left(0, T, B_{2,1}^{1+\frac{d}{2}}\right)} \\
& \leq c\left(1-e^{-(8 k+2 \gamma) T}\right) \delta M .
\end{aligned}
$$

Similarly the term with $K_{4}$ is bounded by

$$
\begin{aligned}
\int_{0}^{T} \sum_{j \geq-1} 2^{\left(\frac{d}{2}\right) j} & \int_{0}^{s} e^{-(8 k+2 \gamma)(s-\tau)} K_{4} d \tau d s \\
& =c \int_{0}^{T} \sum_{j \geq-1} 2^{\left(1+\frac{d}{2}\right) j} \int_{0}^{s} e^{-(8 k+2 \gamma)(s-\tau)} \sum_{k \geq j-1} 2^{\frac{d}{2} k}\left\|\widetilde{\Delta}_{k} w^{(n+1)}\right\|_{L^{2}}\left\|\Delta_{k} u^{(n)}\right\|_{L^{2}} d \tau d s \\
& \leq\left(c \sum_{j \geq-1} 2^{\left(1+\frac{d}{2}\right) j} \int_{0}^{T} \sum_{k \geq j-1} 2^{\frac{d}{2} k}\left\|\widetilde{\Delta}_{k} w^{(n+1)}\right\|_{L^{2}}\left\|\Delta_{k} u^{(n)}\right\|_{L^{2}} d \tau\right)\left(\int_{0}^{T} e^{-(8 k+2 \gamma) s} d s\right)
\end{aligned}
$$




$$
\begin{aligned}
& \leq c\left(1-e^{-(8 k+2 \gamma) T}\right) \int_{0}^{T} \sum_{j \geq-1} 2^{\left(1+\frac{d}{2}\right) j} \sum_{k \geq j-1} 2^{\frac{d}{2} k}\left\|\widetilde{\Delta}_{k} w^{(n+1)}\right\|_{L^{2}}\left\|\Delta_{k} u^{(n)}\right\|_{L^{2}} d \tau \\
& \leq c\left(1-e^{-(8 k+2 \gamma) T}\right) \int_{0}^{T} \sum_{j \geq-1} 2^{\left(1+\frac{d}{2}\right) j}\left\|\Delta_{j} u^{(n)}\right\|_{L^{2}} \sum_{j \geq-1} 2^{\frac{d}{2} j}\left\|\widetilde{\Delta}_{j} w^{(n+1)}\right\|_{L^{2}} d \tau \\
& \leq c\left(1-e^{-(8 k+2 \gamma) T}\right)\left\|w^{(n+1)}\right\|_{L^{\infty}\left(0, T, B_{2,1}^{\frac{d}{2}}\right)}\left\|u^{(n)}\right\|_{L^{1}\left(0, T, B_{2,1}^{1+\frac{d}{2}}\right)} \\
& \leq c\left(1-e^{-(8 k+2 \gamma) T}\right) \delta M .
\end{aligned}
$$

Collecting the estimates above and inserting them in (4.11), we obtain

$$
\left\|w^{(n+1)}\right\|_{L^{1}\left(0, T, B_{2,1}^{\frac{d}{2}}\right)} \leq \frac{\delta}{2}+c\left(1-e^{-(8 k+2 \gamma) T}\right) \delta+c\left(1-e^{-(8 k+2 \gamma) T}\right) \delta M .
$$

Choosing T sufficiently small such that $c\left(1-e^{-(8 k+2 \gamma) T}\right) \leq \min \left(\frac{1}{4 M}, \frac{1}{4}\right)$, we get

$$
\left\|w^{(n+1)}\right\|_{L^{1}\left(0, T, B_{2,1}^{\frac{d}{2}}\right)} \leq \frac{\delta}{2}+\frac{\delta}{4}+\frac{\delta}{4}=\delta .
$$

These uniform bounds allow us to extract a weakly convergent subsequence. That is there is $(u, w) \in Y$ such that a subsequence of $\left(u^{n}, w^{n}\right)$ (still denoted by $\left.\left(u^{n}, w^{n}\right)\right)$ satisfies

$$
\begin{aligned}
& u^{n} \stackrel{*}{\rightarrow} u \quad \text { in } \quad L^{\infty}\left(0, T, B_{2,1}^{1+\frac{d}{2}-2 \alpha}\right), \\
& w^{n} \stackrel{*}{\rightarrow} w \quad \text { in } \quad L^{\infty}\left(0, T, B_{2,1}^{\frac{d}{2}}\right) .
\end{aligned}
$$

In order to show that $(u, w)$ is a weak solution of (4.1) we need to further extract a subsequence which converges strongly to $(u, w)$. We use the Aubin-Lions Lemma. We can show by making use of the equation (3.1) that $\left(\partial_{t} u^{n}, \partial_{t} w^{n}\right)$ is uniformly bounded in

$$
\begin{aligned}
& \partial_{t} u^{n} \in L^{1}\left(0, T, B_{2,1}^{1+\frac{d}{2}-2 \alpha}\right) \cap L^{2}\left(0, T, B_{2,1}^{1+\frac{d}{2}-3 \alpha}\right), \\
& \partial_{t} w^{n} \in L^{1}\left(0, T, B_{2,1}^{\frac{d}{2}}\right) \cap L^{2}\left(0, T, B_{2,1}^{\frac{d}{2}}\right) .
\end{aligned}
$$

Since we are in this case in the whole space $\mathbb{R}^{d}$, we need to combine Cantor's diagonal process with the Aubin-Lions Lemma to show that a subsequence of a weakly convergent subsequence, still denoted by $\left(u^{n}, w^{n}\right)$, has the following strongly convergent property

$$
\left(u^{n}, w^{n}\right) \longrightarrow(u, w) \text { in } \quad L^{2}\left(0, T, B_{2,1}^{1+\frac{d}{2}-\gamma}(Q)\right)
$$

where $\alpha \leq \gamma \leq 3 \alpha$ and $Q \subset \mathbb{R}^{d}$ is a compact subset. This strong convergence property would allow us to show that $(u, w)$ is indeed a weak solution of (4.1). This completes the proof for the existence part of Theorem 1.2. 


\subsection{Uniqueness of weak solutions}

Proof. Assume that $\left(u^{(1)}, w^{(1)}\right)$ and $\left(u^{(2)}, w^{(2)}\right)$ are two solutions of (4.1) in the regularity class in (1.4) and (1.5). Their difference $(\widetilde{u}, \widetilde{w})$ with

$$
\widetilde{u}=u^{(2)}-u^{(1)} \text { and } \widetilde{w}=w^{(2)}-w^{(1)}
$$

satisfies

$$
\left\{\begin{array}{l}
\partial_{t} \widetilde{u}+(\nu+k)(-\Delta)^{\alpha} \widetilde{u}=-\mathbb{P}\left(u^{(2)} \cdot \nabla \widetilde{u}+\widetilde{u} \cdot \nabla u^{(1)}\right)+2 k \nabla \times \widetilde{w} \\
\partial_{t} \widetilde{w}=-(4 k+\gamma) \widetilde{w}-2 k \nabla \times \widetilde{u}-u^{(2)} \cdot \nabla \widetilde{w}-\widetilde{u} \cdot \nabla w^{(1)} \\
\nabla \cdot \widetilde{u}=0, \\
\widetilde{u}(x, 0)=0, \quad \widetilde{w}(x, 0)=0 .
\end{array}\right.
$$

We estimate the difference $(\widetilde{u}, \widetilde{w})$ in $L^{2}\left(\mathbb{R}^{d}\right)$. Dotting $(4.12)$ by $(\widetilde{u}, \widetilde{w})$ and applying the divergence-free condition, we find

$$
\begin{aligned}
\frac{1}{2} \frac{d}{d t} & \left(\|\widetilde{u}\|_{L^{2}}^{2}+\|\widetilde{w}\|_{L^{2}}^{2}\right)+(\nu+k)\left\|\Lambda^{\alpha} \widetilde{u}\right\|_{L^{2}}^{2}+(4 k+\gamma)\|\widetilde{w}\|_{L^{2}}^{2} \\
= & -\int u^{(2)} \cdot \nabla \widetilde{u} \cdot \widetilde{u} d x-\int \widetilde{u} \cdot \nabla u^{(1)} \cdot \widetilde{u} d x \\
& -\int u^{(2)} \cdot \nabla \widetilde{w} \cdot \widetilde{w} d x-\int \widetilde{u} \cdot \nabla w^{(1)} \cdot \widetilde{w} d x \\
= & L_{1}+L_{2}+L_{3}+L_{4},
\end{aligned}
$$

where

$$
\begin{aligned}
& L_{1}=-\int u^{(2)} \cdot \nabla \widetilde{u} \cdot \widetilde{u} d x, \\
& L_{2}=-\int \widetilde{u} \cdot \nabla u^{(1)} \cdot \widetilde{u} d x, \\
& L_{3}=-\int u^{(2)} \cdot \nabla \widetilde{w} \cdot \widetilde{w} d x, \\
& L_{4}=-\int \widetilde{u} \cdot \nabla w^{(1)} \cdot \widetilde{w} d x .
\end{aligned}
$$

Due to $\nabla \cdot u^{(2)}=0$, we find $L_{1}=L_{3}=0$ after integration by parts. As in (3.16),

$$
\left|L_{2}\right| \leq c\left\|u^{(1)}\right\|_{B_{2,1}^{1+\frac{d}{2}}}\|\widetilde{u}\|_{L^{2}}^{2} .
$$

To bound $L_{4}$, We set

$$
\frac{1}{p}=\frac{1}{2}-\frac{\alpha}{d}, \quad \frac{1}{q}=\frac{\alpha}{d} \quad\left(\text { or } \frac{d}{q}=\alpha\right) .
$$


By Hölder's inequality,

$$
\begin{aligned}
& \left|L_{4}\right|=\left|-\int \widetilde{u} \cdot \nabla w^{(1)} \cdot \widetilde{w} d x\right| \\
& \leq\|\widetilde{w}\|_{L^{2}}\left\|\nabla w^{(1)}\right\|_{L^{q}}\|\widetilde{u}\|_{L^{p}} \\
& \leq \sum_{j \geq-1}\left\|\Delta_{j} \nabla w^{(1)}\right\|_{L^{q}}\|\widetilde{w}\|_{L^{2}}\|\widetilde{u}\|_{L^{p}} \\
& \leq c \sum_{j \geq-1} 2^{j} 2^{d j\left(\frac{1}{2}-\frac{1}{q}\right)}\left\|\Delta_{j} w^{(1)}\right\|_{L^{2}}\|\widetilde{w}\|_{L^{2}}\|\widetilde{u}\|_{L^{p}} \\
& =\sum_{j \geq-1} 2^{j} 2^{\frac{d j}{2}-\frac{d}{q} j}\left\|\Delta_{j} w^{(1)}\right\|\left\|_{L^{2}}\right\| \widetilde{w}\left\|_{L^{2}}\right\| \widetilde{u} \|_{L^{p}} \\
& \underset{\text { since } \alpha \geq 1}{\leq} c \sum_{j \geq-1} 2^{\frac{d j}{2}}\left\|\Delta_{j} w^{(1)}\right\|_{L^{2}}\|\widetilde{w}\|_{L^{2}}\|\widetilde{u}\|_{L^{p}} \\
& \leq c\left\|w^{(1)}\right\|_{B_{2,1}^{\frac{d}{2}}}\|\widetilde{w}\|_{L^{2}}\left\|\Lambda^{\alpha} \widetilde{u}\right\|_{L^{2}} \\
& \leq \frac{(\nu+k)}{2}\left\|\Lambda^{\alpha} \widetilde{u}\right\|_{L^{2}}^{2}+c\left\|w^{(1)}\right\|_{B_{2,1}^{\frac{d}{2}}}^{2}\|\widetilde{w}\|_{L^{2}}^{2},
\end{aligned}
$$

Where in the last inequality we make use of

$$
\|\widetilde{u}\|_{L^{p}} \leq c\left\|\Lambda^{\alpha} \widetilde{u}\right\|_{L^{2}}
$$

Combining the estimates leads to

$$
\begin{aligned}
\frac{d}{d t}\left(\|\widetilde{u}\|_{L^{2}}^{2}\right. & \left.+\|\widetilde{w}\|_{L^{2}}^{2}\right)+(\nu+k)\left\|\Lambda^{\alpha} \widetilde{u}\right\|_{L^{2}}^{2}+(8 k+2 \gamma)\|\widetilde{w}\|_{L^{2}}^{2} \\
& \leq\left(c\left\|u^{(1)}\right\|_{B_{2,1}^{1+\frac{d}{2}}}+c\left\|w^{(1)}\right\|_{B_{2,1}^{\frac{d}{2}}}^{2}\right)\left(\|\widetilde{u}\|_{L^{2}}^{2}+\|\widetilde{w}\|_{L^{2}}^{2}\right) .
\end{aligned}
$$

Since $u^{(1)} \in L^{1}\left(0, T, B_{2,1}^{1+\frac{d}{2}}\right)$ and $w^{(1)} \in L^{1}\left(0, T, B_{2,1}^{\frac{d}{2}}\right) \cap L^{\infty}\left(0, T, B_{2,1}^{\frac{d}{2}}\right)$,

$$
\int_{0}^{T}\left\|u^{(1)}(t)\right\|_{B_{2,1}^{1+\frac{d}{2}}} d t<\infty \quad \text { and } \quad \int_{0}^{T}\left\|w^{(1)}(t)\right\|_{B_{2,1}^{\frac{d}{2}}}^{2} d t \leq T\left\|w^{(1)}(t)\right\|_{L^{\infty}\left(0, T, B_{2,1}^{2}\right)}^{2} .
$$

Applying Gronwall's inequality to (4.13) yields

$$
\|\widetilde{u}\|_{L^{2}}=\|\widetilde{w}\|_{L^{2}}=0
$$

which leads to the desired uniqueness. This completes the proof of the uniqueness part of Theorem 1.2.

\section{References}

[1] H. Bahouri, J.-Y. Chemin, R. Danchin, Fourier Analysis and Nonlinear Partial Differential Equations, in: Grundlehren der Mathematischen Wissenschaften, vol. 343, Springer, Heidelberg, 2011. 
[2] J. Boldrini, M. Durán, M. Rojas-Medar, Existence and uniqueness of strong solution for the incompressible micropolar fluid equations in domains of $\mathbb{R}^{3}$, Ann. Univ. Ferrara, Sez. VII, Sci. Mat. 56 (2010), 37-51.

[3] C. Bucur, E. Valdinoci, Nonlocal Diffusion and Applications, Lecture Notes of the Unione Matematica Italiana, vol. 20, Springer, International Publishing, ISBN 978-3-319-287386, 2016, xii, 155 pp.

[4] J. Chen, Z.-M. Chen, B. Dong, Existence of $H^{2}$-global attractors of two-dimensional micropolar fluid flows, J Math. Anal. Appl., 322 (2006), 512-522.

[5] Q. Chen, C. Miao, Global well-posedness for the micropolar fluid system in critical Besov spaces, J. Differential Equations, 252 (2012), 2698-2724.

[6] S.C. Cowin, Polar fluids, Phys. Fluids, 11 (1968), 1919-1927.

[7] B. Dong, J. Li, J. Wu, Global well-posedness and large-time decay for the 2D micropolar equations, J. Differential Equations, 262, (2017), 3488-3523.

[8] B. Dong, J. Wu, X. Xu, Z. Ye, Global regularity for the 2D micropolar equations with fractional dissipation, Discrete Contin. Dyn. Syst., 38(8) (2018), 4133-4162.

[9] B. Dong, Z. Zhang, Global regularity of the 2D micropolar fluid flows with zero angular viscosity, J. Differential Equations, 249 (2010), 200-213.

[10] M.E. Erdogan, Polar effects in the apparent viscosity of suspension, Rheol. Acta, 9 (1970), 434-438.

[11] A. Eringen, Theory of micropolar fluids, J. Math. Mech., 16 (1966), 1-18.

[12] A.C. Eringen, Micropolar fluids with stretch, Int. J. Engng. Eci., 7 (1969), 115-127.

[13] G. Galdi, S. Rionero, A note on the existence and uniqueness of solutions of the micropolar fluid equations, Internat. J. Engrg. Sci., 15 (1977), 105-108.

[14] Q. Jiu, J. Liu, J. Wu, H. Yu, On the initial- and boundary-value problem for $2 D$ micropolar equations with only angular velocity dissipation, Zeitschrift fur Angewandte Mathematik, und Physik, 68 (2017), 68:107.

[15] Q. Jiu, X. Suo, J. Wu and H. Yu, Unique weak solutions of the magnetohydrodynamic equations with fractional dissipation, submitted for publication.

[16] J. Liu, S. Wang, Initial- and boundary-value problem for 2D micropolar equations without angular viscosity, arXiv:1705.05151 [math.AP]

[17] G. Lukaszewicz, On nonstationary flows of asymmetric fluids, Rend. Accad. Naz. Sci. XL Mem. Mat., 12(1988), 83-97. 
[18] G. Lukaszewicz, On the existence, uniqueness and asymptotic properties for solutions of flows of asymmetric fluids, Rend. Accad. Naz. Sci. XL Mem. Mat., 13(1989), 105-120.

[19] G. Lukaszewicz, Micropolar Fluids. Theory and Applications, Modeling and Simulation in Science, Engineering and Technology, Birkhäuser, Boston, 1999.

[20] A. Majda, A. Bertozzi, Vorticity and Incompressible Flow, Cambridge University Press, Cambridge, 2001.

[21] B. Nowakowski, Large time existence of strong solutions to micropolar equations in cylindrical domains, Nonlinear Analysis: RWA, 14 (2013), 635-660.

[22] M. Rojas-Medar, E. Ortega-Torres, The equations of a viscous asymmetric fluid: An interactive approach, Z. Angew. Math. Mech., 85 (2005), 471-489.

[23] L. Xue, Well posedness and zero microrotation viscosity limit of the 2D micropolar fluid equations, Math. Methods Appl. Sci., 34 (2011), 1760-1777.

[24] N. Yamaguchi, Existence of global strong solution to the micropolar fluid systemin a bounded domain, Math. Methods Appl. Sci., 28 (2005), 1507-1526. 